\title{
The Muds of the Clyde Sea Area. III. Chemical and Physical Conditions; Rate and Nature of Sedimentation; and Fauna.
}

By

\author{
Hilary B. Moore, B.Sc., \\ Assistant Naturalist at the Marine Station, Millport.
}

With 11 Figures in the Text.

A PRELIMINARy survey of the area with regard to the distribution of the total nitrogen and phosphate content of the muds was described in a previous paper (Moore, 5). A number of stations were worked, and these were further examined for nature and distribution of particles, water content, and density, and as a result of this survey certain stations were chosen as suitable for more extensive work. At the same time it became evident that the layers in which the greatest and most important changes were taking place were those within a few centimetres of the surface, and these were therefore studied most intensively.

Among these stations many show very irregular deposition, owing to tidal currents or other causes. At some, conditions appear to be comparatively stable, and a series of these was chosen to illustrate the four chief types of mud found in this area, which are as follows:-

Deep Water Type. This is found in the deepest channels where there is little or no tidal action, a slow rate of sedimentation and very little influence from the land, e.g. Station 7, 110 metres; St. 7b, 166 metres; St. 23,73 metres.

Mid-Loch Type. This is a shallower water type than the preceding, usually in a depth of 45 to 75 metres. There is little tidal action and the small amount of matter received from streams is of very fine grade. Deposition is more rapid, and the mud tends to be softer than in the previous type, e.g. Station 8b, 37 m. ; St. 9, 37 m., Kyles of Bute; St. 11, 73 m., Loch Striven; St. 13, 73 m., Loch Goil ; St. 13a, 82 m. ; St. 15, 58 m. ; St. 15a, 64 m., Loch Long; St. 16a, 26 m., Holy Loch.

Loch Head Type. Here there is practically no tidal action; the depth is from 20 to 25 metres, with the exception of Loch Goil Head where it is deeper. Only small streams enter the lochs, but their influence is most marked on these loch-head stations. The mud is of a more open composition, containing more numerous and larger vegetable remains and sand 
grains, e.g. St. 10, 24 m., Loch Striven; St. 12, 51 m., Loch Goil ; St. 14, 22 m., Loch Long; St. 16, 22 m., Holy Loch ; St. 18, 24 m., Gare Loch.

Sandy Mud Type. Although this work has not been extended to shore sands, some sandy muds have been examined for comparison. These occur chiefly in bays and in parts of channels where there is evidence of strong tidal action, e.g. St. 1b, 25 m., Kames Bay, Cumbrae ; St. 17a, 73 m., Dunoon Basin ; St. 25, 46 m., Barrier Plateau.

A number of stations not described in the previous paper have since been worked and their positions are defined in Table I.

\section{TABLE I.}

\section{Positions of New Stations.*}

St. 1b. Kames Bay, Cumbrae, $25 \mathrm{~m}$.

St. 1c. 100 yards North of Hunterston Perch, Fairlie Sands, $22 \mathrm{~m}$.

St. 7b. $\quad 3.5$ miles West of Garroch Head, $166 \mathrm{~m}$.

St. 17d. Kilchattan Bay, 22 m.

St. 23. South of Little Cumbrae light, West of Ardrossan, $73 \mathrm{~m}$.

St. 25. Midway between Ailsa Craig and Campbelton, $46 \mathrm{~m}$.

\section{Method of Sampling.}

The samples consisted of undisturbed cores of mud $3 \cdot 2 \mathrm{~cm}$. in diameter and up to $40 \mathrm{~cm}$. in length. The sampler (Moore and Neill, 6) collects the cores in glass tubes which are then corked at both ends and taken to the laboratory for examination.

\section{Soluble Salts.}

Under natural conditions the soluble salts leave the mud partly by diffusion into the overlying water via the interstitial water of the mud itself, and partly also by the expression of this interstitial water during the packing of the mud. In these estimations, therefore, wet mud was shaken up with a large amount of sea-water, and the increase in concentration of the various salts in this determined; in the case of phosphate, it being undesirable to extract with a citric acid solution, a sample of the mud which when dry would weigh about $100 \mathrm{gm}$. was rubbed down in a little sea-water until no lumps remained, and was then shaken with 2200 c.c. of sea-water for two hours in an end-over-end shaker. This was then allowed to stand for 24 hours in the dark, and the supernatant water siphoned off for analysis. Shaking for this period was found to remove all the soluble phosphate. The results for the other soluble salts are not yet completed.

* The localities of all other stations referred to are given in Journ. Mar. Biol. Assoc., N.S., Vol. XVI, No. 2, 1930, p. 597. 
For the estimation of soluble phosphates, Atkins' modification of Denigès method (Atkins, 1) was used, the water being passed through a washed Whatman No. 3 filter paper before the estimation. This was found to give identical results with samples cleared by centrifuging.

The results for soluble phosphates are given in Table II, and graphically in Figure 1. St. 11 is in most respects normal, so that the curve shown is

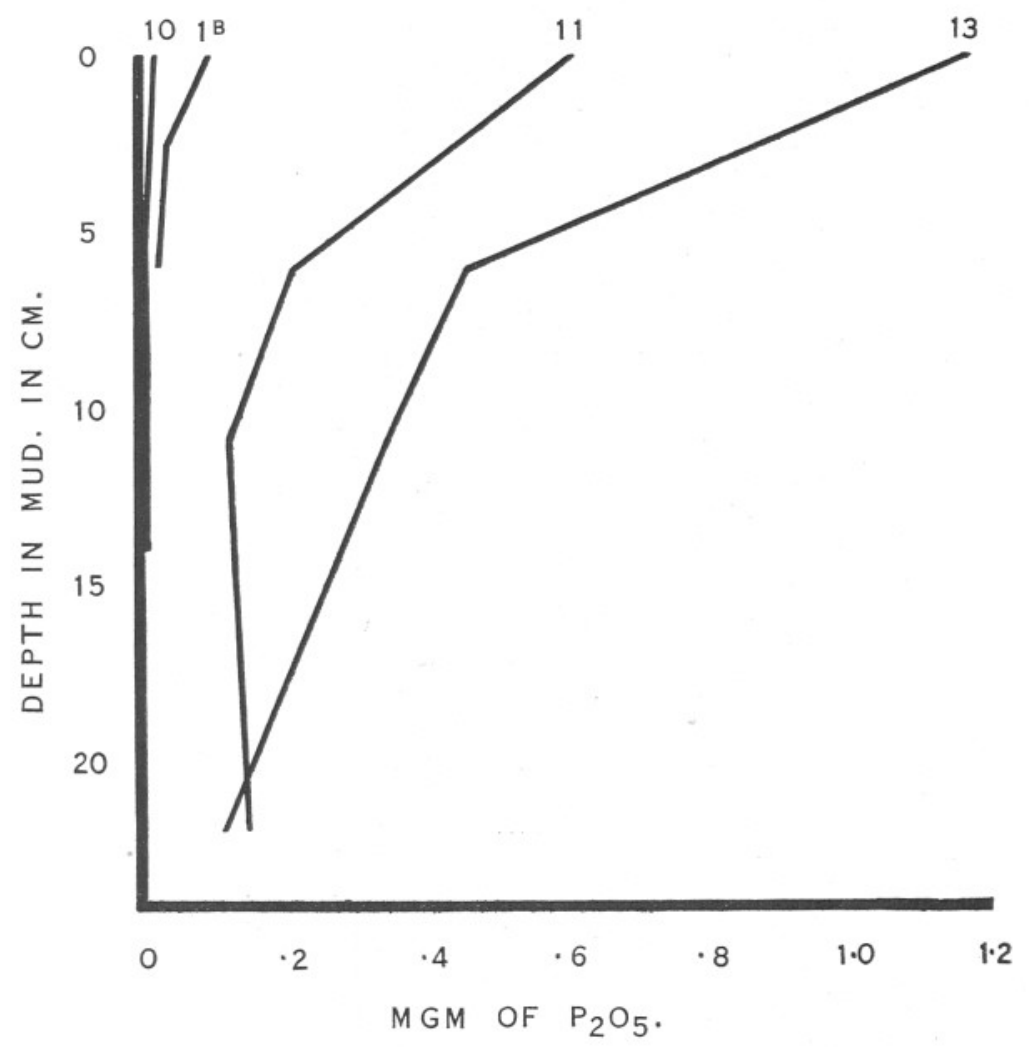

FIG. 1.-Distribution of Soluble Phosphate with depth in the mud.

probably typical for a mid-loch station. It agrees in shape with that for St. 13, except that the latter is much richer in soluble phosphate as it is in other salts including total phosphate. In both, the values are high at the surface of the mud but decrease rapidly to a depth of $6 \mathrm{~cm}$., and then much more slowly. The very low values at St. $1 \mathrm{~b}$ are to be expected in a sandy mud, but the even lower values at St. 10, at Loch Striven Head, are unexpected as the total phosphate there is almost as high as that at St. 11. The more open condition of the mud may allow more rapid loss 
by diffusion, but this does not seem to be sufficient to account for the great difference between the two stations.

It is significant that the greatest loss of soluble phosphate occurs in the same layer as that from which the greatest amount of interstitial water is being expressed by packing, that is the upper $6 \mathrm{~cm}$.

\section{Oxygen Absorption by Mud.}

The condition of the mud with regard to oxygen was estimated by shaking samples of the mud with sea-water of known oxygen content, and measuring the decrease in the latter by Winkler's method. From the

\section{TABLE II.}

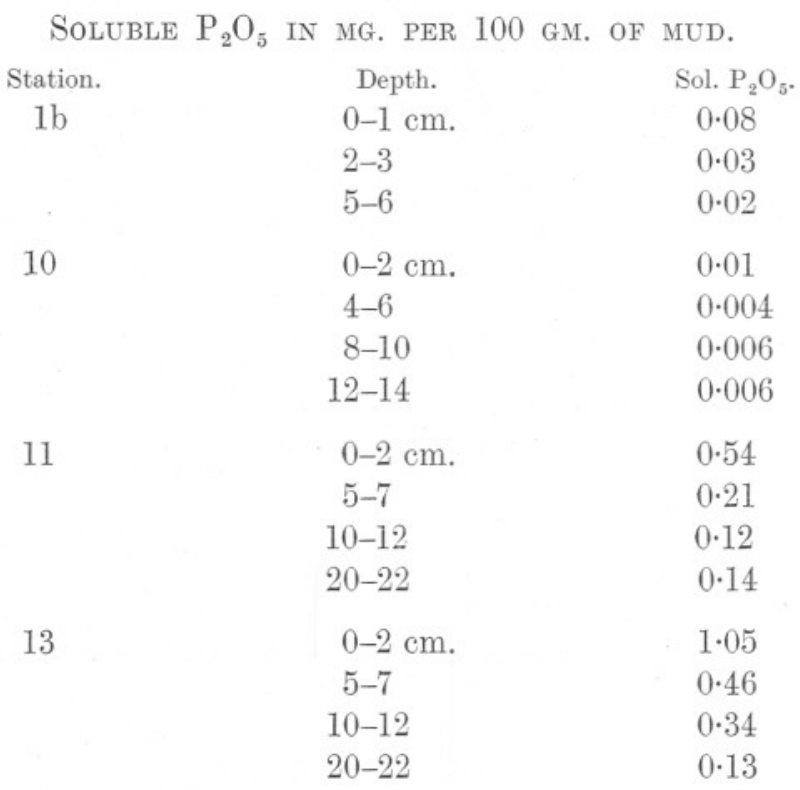

amount taken up in this way it is clear that not only can there be no dissolved oxygen in the interstitial water even at the extreme surface in any mud examined, but also that any oxygen diffusing in from the overlying water will be rapidly absorbed. The general results for all types of station show an increase in the amount of the immediate chemical absorption, as distinct from the slower biological oxygen absorption down to a depth of about $5 \mathrm{~cm}$., below which it becomes more or less constant at about 0.3 to $0.5 \mathrm{mg}$. of oxygen absorbed per gm. of mud (dry weight). In the mid-loch stations where there is a rain of rich organic matter on the surface, the amount of oxygen absorbed by this surface 
layer is correspondingly high, but in the loch head and sandy types the mud is of a more open consistency, allowing deeper diffusion of the overlying water, and this phenomenon is not shown.

\section{ReACTION.}

The estimation of the $\mathrm{pH}$ of the interstitial water of the mud presents certain difficulties; it is not usually possible to withdraw such water except in small quantities and only by the use of reduced pressure on a filter, which would affect the $\mathrm{CO}_{2}$ content and hence the $\mathrm{pH}$. Also turbid solutions may be obtained, so that a colorimetric method of estimation is not reliable.

For the latter reason a quinhydrone electrode was used, with a calomel half-cell as standard (Clark, 2). The soil analysis method of shaking the dried soil with distilled water, is not applicable here, and even shaking the fresh wet mud with distilled water yields erratic results, and always of a low value. On the other hand, if the mud is shaken with sea-water, or with saline solution, regular values are obtained, and these are in agreement with the values obtained from the interstitial water itself in those cases where the mud is of such consistency as to allow of the easy withdrawal of the latter. In practice a tube of mud was cut up into onecentimetre layers, and each of these immediately shaken up with about 20 c.c. of sea-water, the buffering power of the mud being sufficient to overcome that of the water. Quinhydrone was then added to this and the $\mathrm{pH}$ read as quickly as possible. The method is in agreement with that recommended by the International Society of Soil Science $(\mathbf{3})$ with the exception that their suggestion is the use of a solution of $\mathrm{KCl}$ where we have used $\mathrm{NaCl}$. The use of quinhydrone in the presence of a reducing medium such as a mud extract necessarily introduces an error, but the rate of potential drift suggests that this should be small if the estimation is made quickly after the addition of the quinhydrone.

The results for the four types of station are shown in Figure 2. In general, with increasing depth in the mud there is a steady rise in $\mathrm{pH}$ followed by a less rapid fall again, the depth at which the maximum occurs varying from one station to another. The overlying water has a higher $\mathrm{pH}$ than the mud, and its influence seems to affect the top centimetre layer of the mud whose $\mathrm{pH}$ is usually distinctly higher than that of the succeeding centimetre. In the case of the more open sandy and loch-head types, this influence is also felt in the second centimetre. The absence of the rise in the mud from St. 13 is probably due to experimental error, it being very difficult to isolate the semi-fluid surface layer in this case. The drop at 5 to $6 \mathrm{~cm}$. at St. $11 \mathrm{in}$ Loch Striven is a peculiarity of that loch [vide distribution of total $\mathrm{P}_{2} \mathrm{O}_{5}$ (Moore, 5 )], as it occurs in most parts of the channel but not in other lochs. 
Considering the high organic content of these muds it is surprising that the $\mathrm{pH}$ values are so high, the lowest recorded being only $7 \cdot 45$. Curiously, too, a mud as rich as that at St. 13 may have as high a $\mathrm{pH}$ as 7.84 well

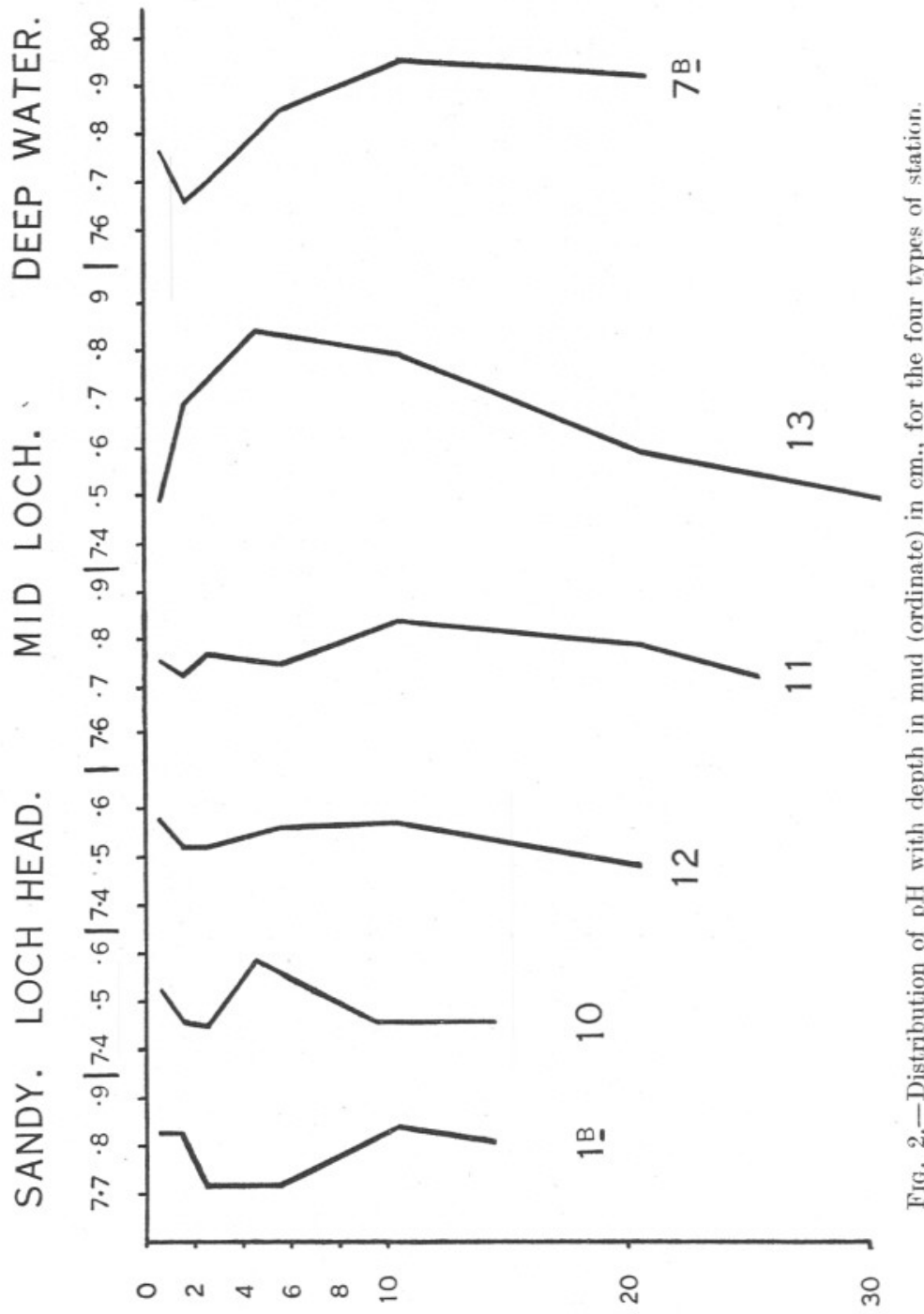

below the surface. The higher surface values are probably to be explained by the influence of the sea-water which has always a higher value than that of the mud. The rise in $\mathrm{pH}$ from $2 \mathrm{~cm}$. downwards may be due to the decreasing amount of organic matter with depth, but this gives no account 
of the succeeding drop which is so marked in the deepest samples taken, i.e. St. 11 and 13. From a biological aspect, however, the outstanding point is the absence of any extremes of $\mathrm{pH}$ even in very different types of mud. The actual values obtained are shown in Table III.

TABLE III.

Distribution of pH with Depth in Mud.

$\begin{array}{ccccccc}\begin{array}{c}\text { Depth in. Mud } \\ \text { in cm. }\end{array} & \text { lb. } & \text { 10. } & \text { 12. } & \text { Stations. } & 13 . & 7 \mathrm{~b} . \\ 0-1 & 7 \cdot 83 & 7 \cdot 53 & 7 \cdot 58 & 7 \cdot 76 & 7 \cdot 49 & 7 \cdot 76 \\ 1-2 & 7 \cdot 83 & 7 \cdot 46 & 7 \cdot 52 & 7 \cdot 73 & 7 \cdot 69 & 7 \cdot 66 \\ 2-3 & 7 \cdot 72 & 7 \cdot 45 & 7 \cdot 52 & 7 \cdot 77 & - & 7 \cdot 70 \\ 4-5 & - & 7 \cdot 59 & - & - & 7 \cdot 84 & - \\ 5-6 & 7 \cdot 72 & - & 7 \cdot 56 & 7 \cdot 75 & - & 7 \cdot 85 \\ 9-10 & - & 7 \cdot 46 & - & - & - & - \\ 10-11 & 7 \cdot 84 & - & 7 \cdot 57 & 7 \cdot 84 & 7 \cdot 79 & 7 \cdot 95 \\ 14-15 & 7 \cdot 81 & 7 \cdot 46 & - & - & - & - \\ 20-21 & - & - & 7 \cdot 48 & 7 \cdot 79 & 7 \cdot 59 & 7 \cdot 92 \\ 25-26 & - & - & - & 7 \cdot 72 & - & - \\ 30-31 & - & - & - & - & 7 \cdot 49 & -\end{array}$

\section{Water Content.}

The distribution of density and water content with depth in the mud was estimated simultaneously in a number of muds. The method found to be most applicable was that of weighing about $10 \mathrm{gm}$. of the wet mud, on a tared watch-glass, then reweighing immersed in sea-water, and finally tipping off the free water from the glass, drying in a water oven at $100^{\circ} \mathrm{C}$., and weighing again. From these results the density of the wet and of the dried mud, and the percentage by volume of water in the wet mud can be calculated. The chief error introduced is that of the salt content of the interstitial water, and of such water as clings to the mud and the watchglass on drying. This will cause the figures for density to be about $5 \%$ too high, but since the water content does not vary sufficiently to affect this appreciably, the error has been assumed to be constant, and no correction has been made for it. Similarly, the figure for the percentage of contained water will be about $2 \%$ too low and will also have an almost constant error. The error due to weighing in sea-water instead of fresh is allowed for.

The values obtained for the various stations, which are expressed as percentages by volume of water in the wet mud, are given in Table IV. It must be understood that these figures do not refer to the amount of true free interstitial water, but to the total loss on drying at $100^{\circ} \mathrm{C}$., some of 
which may be due to water contained in the form of gels or otherwise fixed.

Figure 3 shows the distribution of the water content with depth in the mud for St. 11, a mid-loch type. Here there is a rapid fall in the quantity of water from $85 \%$ at the surface to $79 \%$ at about $6 \mathrm{~cm}$. Below this depth the fall is very much slower. Though the slope of the curve varies at different stations, there occurs typically a rapid fall in water content near the surface followed at a varying depth by a more slowly decreasing or constant amount. The depth at which this constant amount is attained

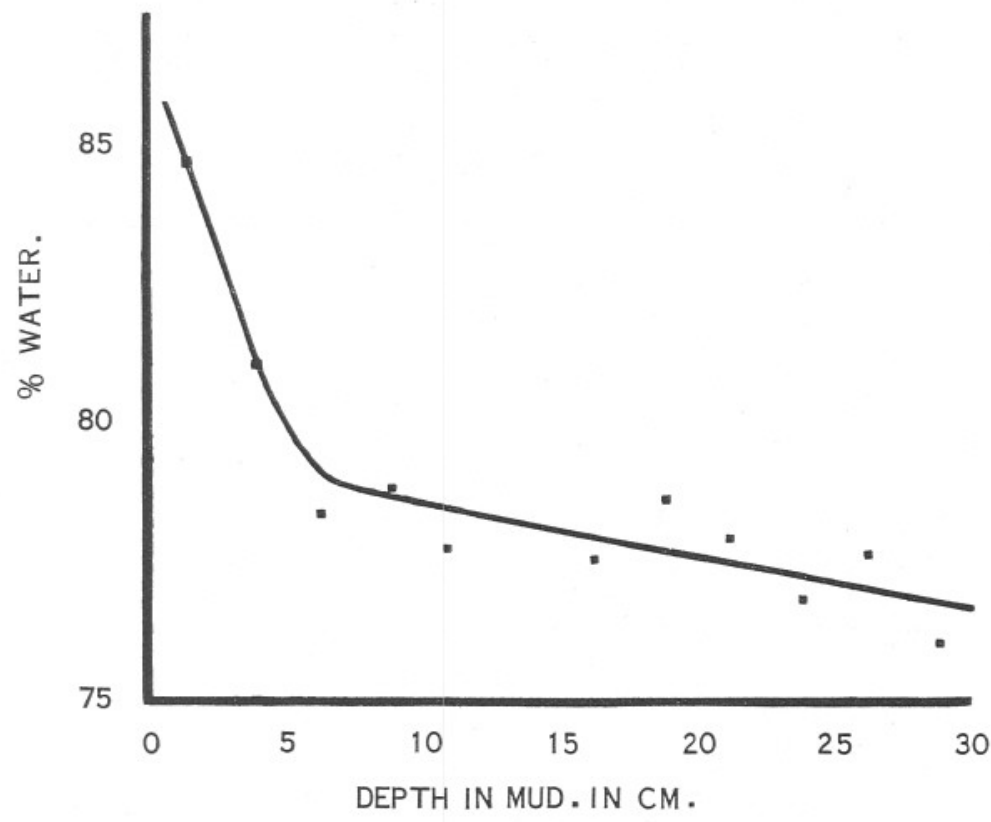

FIG. 3.-Variation of water content with depth in mud at St. 11-mid-loch type.

varies, and in some cases, i.e. St. 26 , the sampling seems not to have been taken deep enough to reach this level, though it would probably be reached with a longer sample. Certain stations are very erratic, notably those in the Dunoon Basin, and of these St. 20a is of interest since a sample there shows at the surface a layer of sand, superimposed at a depth of about $10 \mathrm{~cm}$. on a layer of mud and this again at about $20 \mathrm{~cm}$. on another layer of sand. The water content shows corresponding changes being lower in the sandy layers than in the mud. Since this station must be largely influenced by the river, it would appear that the nature of the material deposited had changed considerably from time to time.

There does not appear to be any correlation between the shape of the curve and the type of station, with the exception of the sandy type which 
has a very much lower water content than the softer muds. In the other types the surface values are remarkably high, frequently lying between $80 \%$ and $85 \%$. The constant value attained usually lies between $70 \%$ and $80 \%$, though in the case of St. 26 the value has fallen from $72 \%$ to $56 \%$ without becoming constant. This station, however, is far from land and of a different type from that of the lochs.

In a later section it is shown that the mud is laid down on the surface

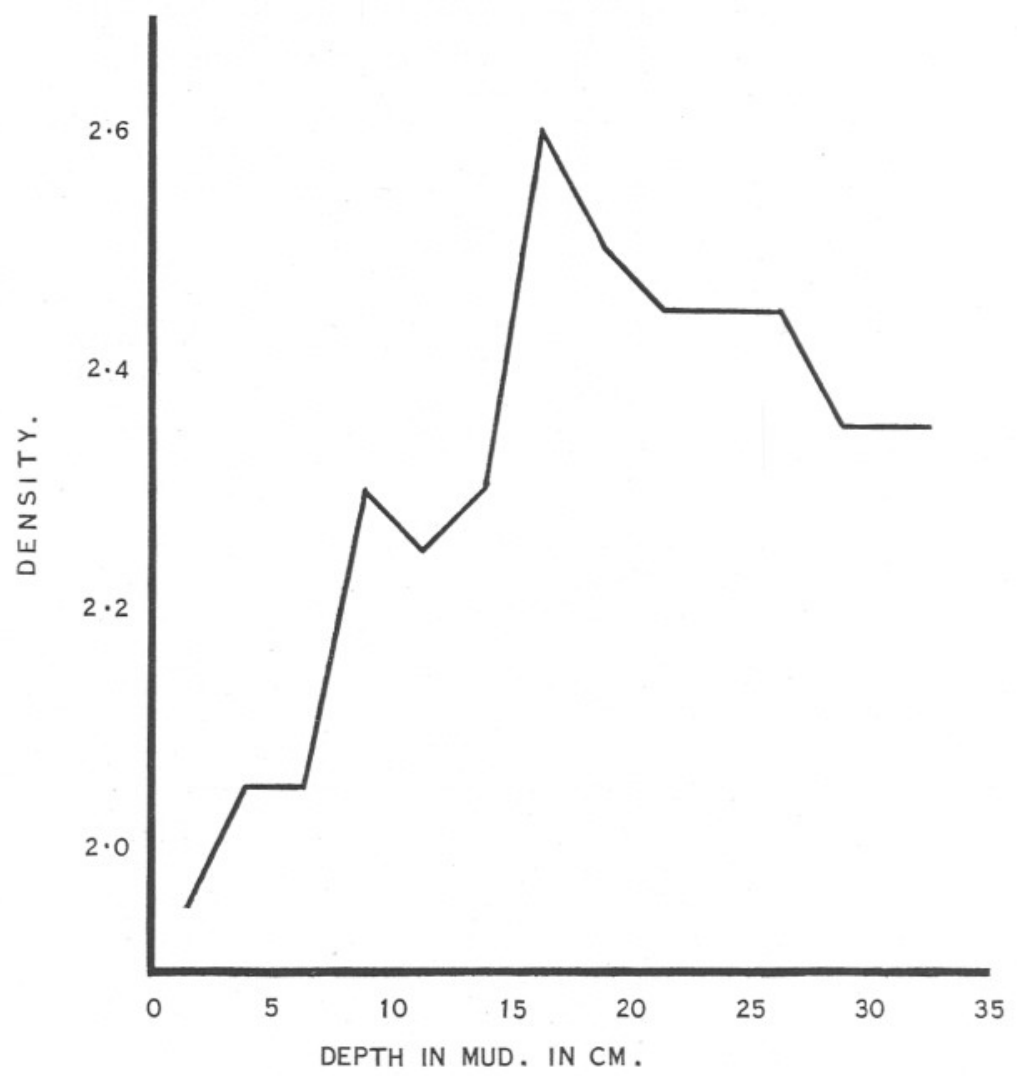

FIG. 4.-Distribution of density with depth in the mud for St. 13-mid-loch type.

in a very fine and loose condition; and an examination of a tube sample shows that it becomes steadily harder and more like a clay with increasing depth. The above figures show that this is due to packing accompanied by expression of water. The interesting point, however, is that this packing continues for such a long time; in the case of St. 11, as shown later, the chief packing being completed at the end of about ten years, and at a depth of about $5 \mathrm{~cm}$., while in cases such as St. 26 packing probably continues for a very much longer period. 
Finally, the cause of the packing may be assigned to several factors. Primarily, no doubt, it is due to the weight of the superimposed mud, but another factor of importance will be the packing effect of the small organisms, such as worms, moving about in the mud ; while yet another factor of great importance is the consolidation of the fine mud particles into firm pellets in the guts of animals which eat them. This question of pellet formation, which is of tremendous importance in some muds, is discussed more fully later. Finally, there is the possibility of packing accompanying chemical change in the break down of organic matter in the mud.

\section{Density.}

The distribution of the density of the dried mud throughout the area is given in Table V, while that for St. 13 is shown graphically in Figure 4, this being fairly typical for the area. Of the nineteen stations worked there was a rise in density below the surface in twelve, followed by a fall in density in half of these. While the density of individual particles may vary much more widely, that of the mud en masse lies usually between $2 \cdot 0$ and 2.5 . These values, together with an increasing density at greater depths, are to be expected in a mud consisting chiefly of a mixture of quartz and calcareous particles with a varying amount of lighter organic matter, the quantity of the latter decreasing with depth.

The subsequent fall at some stations is less clear, especially as it occurs only in the mid-loch type of station where conditions are stable. Of the loch-head type, three stations show a rise but no fall, possibly because the sampling has not been deep enough, the rate of deposition being greater in these. The stations in the Dunoon Basin-Stations 20a, 17, 17a, 17b, $17 \mathrm{c}$-are usually erratic in every way, St. $20 \mathrm{a}$ reflecting its alternate layers of mud and sand in its density. In the sandy type of station there is very little variation, the density being that of the quartz of which it is almost entirely composed.

\section{Nature and Distribution of Particles.}

The abundance of fæcal pellets and the large quantity of organic matter present render sieving an unsuitable method of grading; for the same reason the standard method of grading by rate of settling in distilled water after first drying, treating with $\mathrm{H}_{2} \mathrm{O}_{2}$ and washing with distilled water, would completely alter the nature of the mud from a biological standpoint. An attempt to estimate particle size from rate of settling of fresh mud in sea-water would also yield erroneous results, since the coarser grades may consist of a mixture of quartz grains with pellets of twice their size, while in the finer grades it has been shown that the density of the particles may vary in extreme cases from $1 \cdot 2$ to over $4 \cdot 0$. 
TABLE V.

Distribution of Density with Depth in Mud.

Depth in mud in $\mathrm{cm}$.

\begin{tabular}{|c|c|c|c|c|c|c|c|c|c|c|c|c|c|c|}
\hline 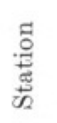 & & $\begin{array}{l}\dot{a} \\
\dot{a} \\
0\end{array}$ & 官 & 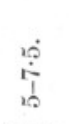 & $\stackrel{\dot{\hat{I}}}{\stackrel{1}{\circ}}$ & 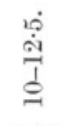 & 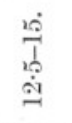 & 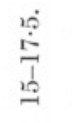 & $\begin{array}{l}\stackrel{A}{1} \\
1 \\
\stackrel{1}{-}\end{array}$ & 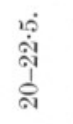 & 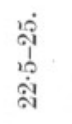 & 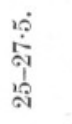 & 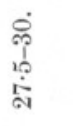 & 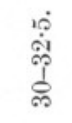 \\
\hline 1 & . & $2 \cdot 60$ & $2 \cdot 60$ & $2 \cdot 60$ & $2 \cdot 60$ & $2 \cdot 60$ & $2 \cdot 65$ & - & - & - & - & - & - & - \\
\hline 11 & . & . $2 \cdot 30$ & $2 \cdot 35$ & $2 \cdot 35$ & $2 \cdot 40$ & $2 \cdot 30$ & $2 \cdot 35$ & $2 \cdot 40$ & $2 \cdot 50$ & $2 \cdot 35$ & $2 \cdot 30$ & $2 \cdot 35$ & $2 \cdot 30$ & - \\
\hline $11 b$ & . & . $2 \cdot 35$ & $2 \cdot 45$ & $2 \cdot 50$ & $2 \cdot 50$ & $2 \cdot 45$ & $2 \cdot 45$ & $2 \cdot 60$ & $2 \cdot 60$ & $2 \cdot 65$ & $2 \cdot 55$ & $2 \cdot 55$ & $2 \cdot 50$ & $2 \cdot 40$ \\
\hline 12 & . & . $2 \cdot 00$ & $2 \cdot 20$ & $2 \cdot 25$ & $2 \cdot 30$ & $2 \cdot 30$ & $2 \cdot 35$ & - & $2 \cdot 20$ & $2 \cdot 25$ & $2 \cdot 30$ & $2 \cdot 25$ & - & - \\
\hline 13 & . & . 1.95 & $2 \cdot 05$ & $2 \cdot 05$ & $2 \cdot 30$ & $2 \cdot 25$ & $2 \cdot 30$ & $2 \cdot 60$ & $2 \cdot 50$ & $2 \cdot 45$ & $2 \cdot 45$ & $2 \cdot 45$ & $2 \cdot 35$ & $2 \cdot 35$ \\
\hline $13 a$ & . & $2 \cdot 30$ & $2 \cdot 20$ & $2 \cdot 40$ & $2 \cdot 45$ & $2 \cdot 45$ & $2 \cdot 45$ & $2 \cdot 40$ & $2 \cdot 50$ & $2 \cdot 50$ & $2 \cdot 25$ & - & - & - \\
\hline 14 & . & $2 \cdot 25$ & $2 \cdot 35$ & $2 \cdot 35$ & $2 \cdot 55$ & $2 \cdot 30$ & $2 \cdot 35$ & $2 \cdot 40$ & $2 \cdot 40$ & $2 \cdot 45$ & $2 \cdot 35$ & $2 \cdot 30$ & $2 \cdot 40$ & - \\
\hline $15 \mathrm{a}$ & . & $2 \cdot 40$ & $2 \cdot 45$ & $2 \cdot 50$ & $2 \cdot 45$ & $2 \cdot 45$ & 2.50 & $2 \cdot 55$ & $2 \cdot 60$ & $2 \cdot 50$ & $2 \cdot 55$ & $2 \cdot 60$ & $2 \cdot 55$ & - \\
\hline 16 & . & . $2 \cdot 35$ & $2 \cdot 40$ & $2 \cdot 45$ & $2 \cdot 40$ & $2 \cdot 45$ & $2 \cdot 40$ & $2 \cdot 35$ & $2 \cdot 50$ & 2.55 & - & - & - & - \\
\hline $16 a$ & . & . 2.40 & $2 \cdot 45$ & $2 \cdot 45$ & $2 \cdot 50$ & $2 \cdot 50$ & $2 \cdot 55$ & $2 \cdot 55$ & $2 \cdot 55$ & $2 \cdot 55$ & $2 \cdot 55$ & $2 \cdot 60$ & $2 \cdot 60$ & - \\
\hline 17 & . & $2 \cdot 50$ & $2 \cdot 45$ & $2 \cdot 40$ & $2 \cdot 45$ & $2 \cdot 40$ & $2 \cdot 50$ & $2 \cdot 40$ & $2 \cdot 45$ & $2 \cdot 45$ & $2 \cdot 45$ & - & - & - \\
\hline $17 \mathrm{a}$ & . & $2 \cdot 65$ & $2 \cdot 60$ & $2 \cdot 65$ & $2 \cdot 65$ & $2 \cdot 65$ & $2 \cdot 65$ & - & - & - & - & - & - & - \\
\hline $17 \mathrm{~b}$ & . & $2 \cdot 50$ & $2 \cdot 50$ & $2 \cdot 50$ & $2 \cdot 45$ & $2 \cdot 45$ & $2 \cdot 50$ & $2 \cdot 65$ & $2 \cdot 60$ & $2 \cdot 70$ & $2 \cdot 55$ & $2 \cdot 55$ & $2 \cdot 55$ & $2 \cdot 60$ \\
\hline $17 \mathrm{c}$ & . & $2 \cdot 40$ & $2 \cdot 35$ & $2 \cdot 45$ & $2 \cdot 35$ & $2 \cdot 40$ & $2 \cdot 40$ & $2 \cdot 45$ & $2 \cdot 35$ & $2 \cdot 45$ & $2 \cdot 45$ & $2 \cdot 45$ & - & - \\
\hline 19 & . & \multicolumn{2}{|c|}{$2 \cdot 30$} & \multicolumn{2}{|c|}{$2 \cdot 35$} & \multicolumn{2}{|c|}{$2 \cdot 35$} & \multicolumn{2}{|c|}{$2 \cdot 40$} & \multicolumn{2}{|c|}{$2 \cdot 30$} & & & \\
\hline $20 \mathrm{a}$ & . & $2 \cdot 50$ & $2 \cdot 40$ & $2 \cdot 45$ & $2 \cdot 40$ & $2 \cdot 40$ & $2 \cdot 40$ & $2 \cdot 40$ & $2 \cdot 45$ & $2 \cdot 55$ & - & - & - & - \\
\hline 23 & . & \multicolumn{2}{|c|}{$2 \cdot 35$} & \multicolumn{2}{|c|}{$2 \cdot 45$} & \multicolumn{2}{|c|}{$2 \cdot 45$} & \multicolumn{2}{|c|}{$2 \cdot 45$} & \multicolumn{2}{|c|}{$2 \cdot 50$} & \multicolumn{2}{|c|}{$2 \cdot 50$} & \\
\hline 24 & . & $2 \cdot 40$ & $2 \cdot 35$ & $2 \cdot 35$ & $2 \cdot 45$ & $2 \cdot 45$ & $2 \cdot 45$ & $2 \cdot 50$ & $2 \cdot 55$ & $2 \cdot 50$ & $2 \cdot 45$ & $2 \cdot 50$ & $2 \cdot 55$ & - \\
\hline 26 & . & . $2 \cdot 40$ & $2 \cdot 45$ & $2 \cdot 50$ & $2 \cdot 45$ & $2 \cdot 50$ & $2 \cdot 55$ & $2 \cdot 55$ & $2 \cdot 60$ & $2 \cdot 55$ & $2 \cdot 60$ & - & - & - \\
\hline
\end{tabular}


One of the most significant factors in these muds is the conversion into fæcal pellets of the very fine material falling on the surface. In extreme cases the whole of the mud may consist of pellets with no admixture of fine material at all, and it is normal for them to form up to $40 \%$ of the total. For these reasons a simple type of elutriator has been used which differentiates the mud into four grades of fairly definite constitution, and gives an idea of the distribution of the pellets and their relation to the fine material. The elutriation is carried out with sea-water, in four upright conical glass vessels connected in series, the vertical rate of flow at the widest part in each being as follows:-

$\begin{array}{cc}\text { Grade. } & \begin{array}{c}\text { Rate of flow in } \\ \text { cm. per minute. }\end{array} \\ \text { G. } 1 & 10 \cdot 0 \\ \text { G. } 2 & 2 \cdot 5 \\ \text { G. } 3 & 1 \cdot 5 \\ \text { G. } 4 & 1 \cdot 0\end{array}$

The constitution of these grades is fairly constant and the following description of the grades from a bucket sample from St. 10 may be generally applied. Where there are many fragments of plant origin they are distributed in all grades according to size. Occasionally also in the surface layers Grade I may contain numerous very small worm tubes.

Grade I. Chiefly pellets of large Maldanid worms (length 0.2 to $1.0 \mathrm{~mm}$.) and of Syndosmya alba (length ca. $0.5 \mathrm{~mm}$.) ; also some large masses of organic detritus, and sand grains of about $0.1 \mathrm{~mm}$. long. In deep-water stations there are very few large sand grains.

Grade II. Almost entirely pellets of Maldanids (length up to $0.2 \mathrm{~mm}$.), and some small sand grains.

Grade III. Chiefly small mineral fragments, with masses of fine detritus, some recognisably of organic origin, up to $0.1 \mathrm{~mm}$. long, and a few small Maldanid pellets of the same length or less.

Grade IV. Similar to Grade III only of smaller size and with no pellets.

The small amount of material passing Grade IV has been neglected.

Before elutriation it is necessary to rub the mud in some sea-water into a fluid condition. This causes an appreciable amount of break down of the large pellets in Grade I. The surface mud is soft and needs very little rubbing, so that in it there will be little break down, but in the deeper and harder muds more rubbing is necessary, and hence more break down takes place. The pellets in Grade II are smaller, and unless the mud is so stiff as to necessitate considerable rubbing they will be little subject to break down. This is borne out by the fact that in the case of softer muds, such as those from Stations 14 and 16a, the sums of Grades III and IV, into which the pellets would break down, show no steady rise with increasing depth. They do, however, show such a rise in St. 11 which is more clayey. 
TABLE

\section{Elutriation Grade}

Expressed as percentage

\begin{tabular}{|c|c|c|c|c|c|c|c|c|c|c|c|c|c|c|}
\hline$\frac{\dot{\vec{H}}}{\frac{5}{5}}$ & 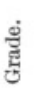 & $\begin{array}{l}\dot{0} \\
\dot{0} \\
0\end{array}$ & $\begin{array}{l}\dot{0} \\
\dot{1} \\
\dot{a} \\
\text { d }\end{array}$ & $\stackrel{\leftrightarrow}{\overrightarrow{1}}$ & $\frac{\dot{\sigma}}{\dot{1}}$ & 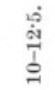 & 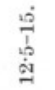 & $\frac{1}{i}$ & 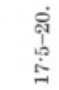 & $\begin{array}{l}\dot{10} \\
\dot{\text { งิ }} \\
\dot{1}\end{array}$ & $\begin{array}{l}\text { â่ } \\
\text { ஸे } \\
\text { ஸे }\end{array}$ & $\begin{array}{l}\dot{0} \\
\stackrel{1}{a} \\
\frac{1}{\omega}\end{array}$ & $\begin{array}{l}\dot{\Phi} \\
\dot{0} \\
\dot{\mathrm{i}}\end{array}$ & $\begin{array}{l}\dot{0} \\
\dot{\phi} \\
\dot{0} \\
0\end{array}$ \\
\hline 1. & $\begin{array}{l}1 . \\
2 . \\
3 . \\
4 .\end{array}$ & $\begin{array}{r}9.2 \\
67.2 \\
0.5 \\
23.1\end{array}$ & $\begin{array}{r}1.1 \\
72.5 \\
0.6 \\
25.7\end{array}$ & $\begin{array}{r}4 \cdot 5 \\
26 \cdot 9 \\
30 \cdot 8 \\
37 \cdot 8\end{array}$ & $\begin{array}{r}2 \cdot 0 \\
69 \cdot 8 \\
0.6 \\
27 \cdot 6\end{array}$ & $\begin{array}{r}1 \cdot 0 \\
64 \cdot 8 \\
14 \cdot 8 \\
19 \cdot 4\end{array}$ & $\begin{array}{r}0 \cdot 9 \\
65 \cdot 2 \\
16 \cdot 4 \\
17 \cdot 2\end{array}$ & & & & & & & \\
\hline $6 a$. & $\begin{array}{l}1 . \\
2 . \\
3 . \\
4 .\end{array}$ & \multicolumn{2}{|c|}{$\begin{array}{l}11 \cdot 7 \\
32 \cdot 1 \\
27 \cdot 9 \\
28 \cdot 3\end{array}$} & \multicolumn{2}{|c|}{$\begin{array}{r}2 \cdot 4 \\
36 \cdot 6 \\
0 \cdot 7 \\
60 \cdot 3\end{array}$} & \multicolumn{2}{|c|}{$\begin{array}{l}12 \cdot 1 \\
15 \cdot 0 \\
43 \cdot 5 \\
29 \cdot 4\end{array}$} & \multicolumn{2}{|c|}{$\begin{array}{r}2 \cdot 7 \\
31 \cdot 4 \\
35 \cdot 2 \\
30 \cdot 8\end{array}$} & & & & & \\
\hline 8. & $\begin{array}{l}1 . \\
2 . \\
3 . \\
4 .\end{array}$ & \multicolumn{2}{|c|}{$\begin{array}{r}8 \cdot 9 \\
43 \cdot 2 \\
5 \cdot 7 \\
42 \cdot 2\end{array}$} & \multicolumn{2}{|c|}{$\begin{array}{r}0 \cdot 9 \\
34 \cdot 1 \\
28 \cdot 1 \\
36 \cdot 9\end{array}$} & \multicolumn{2}{|c|}{$\begin{array}{r}5 \cdot 9 \\
45 \cdot 1 \\
1 \cdot 0 \\
48 \cdot 0\end{array}$} & \multicolumn{2}{|c|}{$\begin{array}{r}2 \cdot 5 \\
62 \cdot 6 \\
25 \cdot 9 \\
9 \cdot 1\end{array}$} & \multicolumn{2}{|c|}{$\begin{array}{r}2 \cdot 2 \\
37 \cdot 9 \\
0 \cdot 1 \\
59 \cdot 8\end{array}$} & & & \\
\hline $8 b$. & $\begin{array}{l}1 . \\
2 . \\
3 . \\
4 .\end{array}$ & \multicolumn{2}{|c|}{$\begin{array}{l}10 \cdot 6 \\
33 \cdot 0 \\
25 \cdot 8 \\
30 \cdot 6\end{array}$} & & & \multicolumn{2}{|c|}{$\begin{array}{l}11 \cdot 3 \\
38 \cdot 6 \\
36 \cdot 9 \\
13 \cdot 2\end{array}$} & \multicolumn{2}{|c|}{$\begin{array}{r}9 \cdot 4 \\
19 \cdot 9 \\
49 \cdot 1 \\
21 \cdot 6\end{array}$} & \multicolumn{2}{|c|}{$\begin{array}{r}0.9 \\
16.2 \\
1.9 \\
81.0\end{array}$} & & & \\
\hline 9. & $\begin{array}{l}1 . \\
2 . \\
3 . \\
4 .\end{array}$ & \multicolumn{2}{|c|}{$\begin{array}{l}16.5 \\
33.7 \\
19 \cdot 6 \\
30 \cdot 2\end{array}$} & \multicolumn{2}{|c|}{$\begin{array}{l}14 \cdot 0 \\
23 \cdot 3 \\
27 \cdot 2 \\
35 \cdot 5\end{array}$} & \multicolumn{2}{|c|}{$\begin{array}{r}2 \cdot 7 \\
33 \cdot 8 \\
0 \cdot 6 \\
62 \cdot 9\end{array}$} & \multicolumn{2}{|c|}{$\begin{array}{r}1.5 \\
29.9 \\
1.7 \\
66.9\end{array}$} & & & & & \\
\hline 11. & $\begin{array}{l}1 . \\
2 . \\
3 . \\
4 .\end{array}$ & $\begin{array}{r}13.0 \\
41.5 \\
1.7 \\
43.8\end{array}$ & $\begin{array}{l}25 \cdot 9 \\
33 \cdot 1 \\
20 \cdot 7 \\
20 \cdot 3\end{array}$ & $\begin{array}{l}14 \cdot 5 \\
41 \cdot 5 \\
17 \cdot 1 \\
26 \cdot 9\end{array}$ & $\begin{array}{r}7.0 \\
46.5 \\
16.5 \\
30 \cdot 0\end{array}$ & & $\begin{array}{r}8 \cdot 1 \\
29 \cdot 1 \\
47 \cdot 1 \\
15 \cdot 7\end{array}$ & $\begin{array}{r}3 \cdot 2 \\
24 \cdot 4 \\
57 \cdot 5 \\
14 \cdot 9\end{array}$ & $\begin{array}{r}4 \cdot 2 \\
13 \cdot 4 \\
69 \cdot 0 \\
13 \cdot 4\end{array}$ & $\begin{array}{r}2 \cdot 1 \\
18 \cdot 4 \\
59 \cdot 0 \\
20 \cdot 5\end{array}$ & $\begin{array}{r}2 \cdot 2 \\
27 \cdot 4 \\
50 \cdot 7 \\
19 \cdot 7\end{array}$ & $\begin{array}{r}3 \cdot 7 \\
24 \cdot 2 \\
52 \cdot 7 \\
19 \cdot 4\end{array}$ & $\begin{array}{r}1.9 \\
15.9 \\
10.0 \\
73.2\end{array}$ & \\
\hline 11b. & $\begin{array}{l}1 . \\
2 . \\
3 . \\
4 .\end{array}$ & $\begin{array}{r}9.0 \\
44.6 \\
12.5 \\
33.9\end{array}$ & $\begin{array}{r}4 \cdot 9 \\
44 \cdot 7 \\
18 \cdot 6 \\
31 \cdot 8\end{array}$ & $\begin{array}{r}1.9 \\
42.5 \\
23.8 \\
31.8\end{array}$ & $\begin{array}{l}13 \cdot 0 \\
30 \cdot 1 \\
24 \cdot 2 \\
32 \cdot 7\end{array}$ & $\begin{array}{r}2 \cdot 4 \\
22 \cdot 7 \\
44 \cdot 2 \\
30 \cdot 7\end{array}$ & $\begin{array}{r}3 \cdot 0 \\
37 \cdot 1 \\
36 \cdot 2 \\
23 \cdot 7\end{array}$ & $\begin{array}{r}3.4 \\
33 \cdot 2 \\
11.6 \\
52 \cdot 0\end{array}$ & $\begin{array}{l}13 \cdot 3 \\
26 \cdot 6 \\
30 \cdot 7 \\
29 \cdot 4\end{array}$ & $\begin{array}{r}1 \cdot 2 \\
25 \cdot 8 \\
42 \cdot 1 \\
30 \cdot 9\end{array}$ & & $\begin{array}{r}2 \cdot 3 \\
32 \cdot 1 \\
2 \cdot 1 \\
63 \cdot 5\end{array}$ & $\begin{array}{r}5.0 \\
39.8 \\
3.0 \\
52.2\end{array}$ & $\begin{array}{r}24 \cdot 3 \\
23 \cdot 5 \\
1.8 \\
50 \cdot 4\end{array}$ \\
\hline 12. & $\begin{array}{l}1 . \\
2 . \\
3 . \\
4 .\end{array}$ & $\begin{array}{r}3.6 \\
32 \cdot 6 \\
0.7 \\
63 \cdot 1\end{array}$ & & $\begin{array}{r}3 \cdot 7 \\
43 \cdot 7 \\
0 \cdot 6 \\
52 \cdot 0\end{array}$ & $\begin{array}{r}3.8 \\
62.4 \\
1.8 \\
32.0\end{array}$ & $\begin{array}{r}2.0 \\
50.2 \\
1.0 \\
46.8\end{array}$ & $\begin{array}{r}2.0 \\
50.3 \\
1.5 \\
46.2\end{array}$ & $\begin{array}{r}0.9 \\
40.3 \\
2.0 \\
56.8\end{array}$ & $\begin{array}{r}0 \cdot 7 \\
20 \cdot 5 \\
65 \cdot 2 \\
13 \cdot 6\end{array}$ & $\begin{array}{r}2 \cdot 7 \\
55 \cdot 3 \\
1 \cdot 4 \\
40 \cdot 6\end{array}$ & $\begin{array}{r}6.9 \\
56 \cdot 0 \\
0.7 \\
36.4\end{array}$ & $\begin{array}{r}4.6 \\
78 \cdot 7 \\
0.7 \\
16.0\end{array}$ & & \\
\hline 13. & $\begin{array}{l}1 . \\
2 . \\
3 . \\
4 .\end{array}$ & $\begin{array}{r}12 \cdot 2 \\
52 \cdot 1 \\
3 \cdot 4 \\
32 \cdot 3\end{array}$ & $\begin{array}{r}2 \cdot 9 \\
57 \cdot 8 \\
3 \cdot 2 \\
36 \cdot 1\end{array}$ & $\begin{array}{r}3 \cdot 7 \\
53 \cdot 7 \\
3 \cdot 4 \\
39 \cdot 2\end{array}$ & $\begin{array}{r}3 \cdot 8 \\
68 \cdot 2 \\
2 \cdot 2 \\
25 \cdot 8\end{array}$ & $\begin{array}{r}3 \cdot 9 \\
59 \cdot 7 \\
15 \cdot 2 \\
21 \cdot 2\end{array}$ & $\begin{array}{r}1 \cdot 3 \\
54 \cdot 1 \\
2 \cdot 0 \\
42 \cdot 6\end{array}$ & $\begin{array}{r}2.1 \\
49.5 \\
21.8 \\
26.6\end{array}$ & $\begin{array}{r}3.6 \\
60.2 \\
3.8 \\
32.4\end{array}$ & $\begin{array}{r}1 \cdot 7 \\
46 \cdot 6 \\
26 \cdot 5 \\
25 \cdot 2\end{array}$ & $\begin{array}{r}1 \cdot 5 \\
47 \cdot 7 \\
20 \cdot 6 \\
30 \cdot 2\end{array}$ & $\begin{array}{r}1 \cdot 7 \\
50 \cdot 1 \\
3 \cdot 4 \\
44 \cdot 8\end{array}$ & $\begin{array}{r}7.4 \\
48.3 \\
1.4 \\
42.9\end{array}$ & $\begin{array}{r}8.9 \\
38.2 \\
1.8 \\
51.1\end{array}$ \\
\hline 13a. & $\begin{array}{l}1 . \\
2 . \\
3 . \\
4 .\end{array}$ & $\begin{array}{r}15 \cdot 2 \\
71 \cdot 3 \\
1.6 \\
11 \cdot 9\end{array}$ & $\begin{array}{r}6 \cdot 3 \\
57 \cdot 7 \\
1 \cdot 2 \\
34 \cdot 8\end{array}$ & $\begin{array}{r}2 \cdot 2 \\
72 \cdot 4 \\
1 \cdot 3 \\
24 \cdot 1\end{array}$ & $\begin{array}{r}9.7 \\
56.8 \\
2.8 \\
30.7\end{array}$ & $\begin{array}{r}1 \cdot 9 \\
41 \cdot 3 \\
29 \cdot 2 \\
27 \cdot 6\end{array}$ & $\begin{array}{r}0 \cdot 9 \\
38 \cdot 7 \\
17 \cdot 7 \\
42 \cdot 7\end{array}$ & $\begin{array}{r}1.6 \\
33.9 \\
38.9 \\
25.6\end{array}$ & $\begin{array}{r}1.5 \\
39 \cdot 9 \\
25 \cdot 3 \\
33.3\end{array}$ & $\begin{array}{r}1.9 \\
40.0 \\
9.7 \\
48.4\end{array}$ & & & & \\
\hline 14. & $\begin{array}{l}1 . \\
2 . \\
3 . \\
4 .\end{array}$ & $\begin{array}{r}6 \cdot 1 \\
52 \cdot 3 \\
1.4 \\
40.3\end{array}$ & $\begin{array}{l}11 \cdot 3 \\
47 \cdot 8 \\
14 \cdot 0 \\
26 \cdot 9\end{array}$ & & $\begin{array}{r}3 \cdot 7 \\
56 \cdot 0 \\
16.8 \\
23.5\end{array}$ & $\begin{array}{r}7 \cdot 2 \\
53 \cdot 4 \\
1 \cdot 3 \\
38 \cdot 1\end{array}$ & $\begin{array}{r}3 \cdot 1 \\
64 \cdot 4 \\
6 \cdot 1 \\
26 \cdot 4\end{array}$ & $\begin{array}{r}10 \cdot 0 \\
53 \cdot 2 \\
1 \cdot 5 \\
35 \cdot 3\end{array}$ & $\begin{array}{r}10.6 \\
56.8 \\
1.9 \\
30.7\end{array}$ & $\begin{array}{r}6 \cdot 4 \\
43 \cdot 6 \\
1.9 \\
48 \cdot 1\end{array}$ & $\begin{array}{r}2 \cdot 1 \\
53 \cdot 3 \\
1 \cdot 3 \\
43 \cdot 3\end{array}$ & $\begin{array}{r}3.5 \\
50.5 \\
2.6 \\
43.4\end{array}$ & $\begin{array}{r}6.2 \\
51.9 \\
1.2 \\
40.7\end{array}$ & \\
\hline 15. & $\begin{array}{l}1 . \\
2 . \\
3 . \\
4 .\end{array}$ & $\begin{array}{r}0.6 \\
65.7 \\
0.9 \\
32.8\end{array}$ & $\begin{array}{r}6.2 \\
58.6 \\
3.4 \\
31.8\end{array}$ & $\begin{array}{r}13 \cdot 7 \\
83 \cdot 0 \\
0 \cdot 4 \\
2 \cdot 9\end{array}$ & $\begin{array}{r}12.5 \\
55.3 \\
0.9 \\
31.3\end{array}$ & $\begin{array}{r}12 \cdot 8 \\
47 \cdot 7 \\
0 \cdot 8 \\
38 \cdot 7\end{array}$ & & $\begin{array}{r}2 \cdot 5 \\
52 \cdot 8 \\
0 \cdot 6 \\
44 \cdot 1\end{array}$ & $\begin{array}{r}2.5 \\
44.2 \\
0.5 \\
52.8\end{array}$ & $\begin{array}{r}3 \cdot 8 \\
46 \cdot 3 \\
0 \cdot 7 \\
49 \cdot 2\end{array}$ & $\begin{array}{r}2 \cdot 2 \\
29 \cdot 3 \\
0 \cdot 4 \\
68 \cdot 1\end{array}$ & $\begin{array}{r}1 \cdot 4 \\
20 \cdot 4 \\
60 \cdot 8 \\
17 \cdot 4\end{array}$ & $\begin{array}{r}7 \cdot 3 \\
16 \cdot 0 \\
70 \cdot 0 \\
6 \cdot 7\end{array}$ & \\
\hline $15 \mathrm{a}$. & $\begin{array}{l}1 . \\
2 . \\
3 . \\
4 .\end{array}$ & $\begin{array}{r}29 \cdot 8 \\
33 \cdot 4 \\
1 \cdot 2 \\
35 \cdot 6\end{array}$ & $\begin{array}{r}21 \cdot 9 \\
61 \cdot 2 \\
1 \cdot 2 \\
15 \cdot 7\end{array}$ & & $\begin{array}{r}2.3 \\
34.9 \\
1.0 \\
61.8\end{array}$ & $\begin{array}{r}2 \cdot 4 \\
30 \cdot 1 \\
30 \cdot 3 \\
37 \cdot 2\end{array}$ & $\begin{array}{r}6 \cdot 4 \\
34 \cdot 5 \\
9 \cdot 5 \\
49 \cdot 6\end{array}$ & $\begin{array}{r}3 \cdot 6 \\
35 \cdot 8 \\
2 \cdot 3 \\
58 \cdot 3\end{array}$ & $\begin{array}{r}3.7 \\
38.8 \\
1.0 \\
56.5\end{array}$ & $\begin{array}{r}5 \cdot 6 \\
31 \cdot 3 \\
28 \cdot 0 \\
35 \cdot 1\end{array}$ & $\begin{array}{r}8 \cdot 3 \\
26 \cdot 8 \\
29 \cdot 6 \\
35 \cdot 3\end{array}$ & $\begin{array}{r}5 \cdot 7 \\
23 \cdot 7 \\
28 \cdot 0 \\
42 \cdot 6\end{array}$ & $\begin{array}{l}11 \cdot 7 \\
15 \cdot 8 \\
38 \cdot 9 \\
33 \cdot 6\end{array}$ & \\
\hline
\end{tabular}


VI.

\section{Percentages.}

dry weight.

\begin{tabular}{|c|c|c|c|c|c|c|c|c|c|c|c|c|c|}
\hline 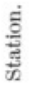 & $\begin{array}{l}\dot{a} \\
\dot{\alpha} \\
0\end{array}$ & $\begin{array}{l}\dot{0} \\
\dot{1} \\
\dot{\omega}\end{array}$ & $\underset{\mid}{\stackrel{1}{\mid c}}$ & 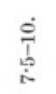 & $\begin{array}{l}\dot{0} \\
\dot{\omega} \\
\hat{1}\end{array}$ & 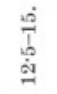 & $\frac{1}{5}$ & $\begin{array}{l}\text { बें } \\
\text { 1े } \\
\stackrel{1}{-1}\end{array}$ & 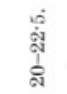 & 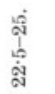 & $\begin{array}{l}\stackrel{\dot{0}}{\text { के }} \\
\text { 守 }\end{array}$ & $\begin{array}{l}\dot{0} \\
0 \\
\dot{0} \\
\dot{1}\end{array}$ & 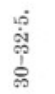 \\
\hline 16. & $\begin{array}{r}3 \cdot 6 \\
45 \cdot 7 \\
1 \cdot 3 \\
49 \cdot 4\end{array}$ & $\begin{array}{r}4 \cdot 9 \\
34.8 \\
0 \cdot 3 \\
60 \cdot 0\end{array}$ & $\begin{array}{r}4 \cdot 2 \\
32 \cdot 9 \\
2 \cdot 3 \\
60 \cdot 6\end{array}$ & $\begin{array}{r}5.6 \\
31 \cdot 1 \\
1.9 \\
61.4\end{array}$ & $\begin{array}{r}5 \cdot 3 \\
36 \cdot 9 \\
37 \cdot 4 \\
20 \cdot 4\end{array}$ & $\begin{array}{r}6.8 \\
39.5 \\
30.8 \\
22.9\end{array}$ & $\begin{array}{r}4 \cdot 7 \\
41 \cdot 5 \\
27 \cdot 1 \\
26 \cdot 7\end{array}$ & $\begin{array}{r}2 \cdot 4 \\
42 \cdot 2 \\
20 \cdot 3 \\
35 \cdot 1\end{array}$ & $\begin{array}{r}2 \cdot 1 \\
43 \cdot 8 \\
5 \cdot 2 \\
48 \cdot 9\end{array}$ & $\begin{array}{r}2 \cdot 4 \\
37 \cdot 7 \\
14 \cdot 2 \\
45 \cdot 7\end{array}$ & & & \\
\hline $16 \mathrm{a}$. & $\begin{array}{r}4.6 \\
45 \cdot 6 \\
1.6 \\
48.2\end{array}$ & $\begin{array}{l}13 \cdot 6 \\
44 \cdot 2 \\
18 \cdot 7 \\
23 \cdot 5\end{array}$ & $\begin{array}{r}6 \cdot 2 \\
37 \cdot 9 \\
30 \cdot 6 \\
25 \cdot 3\end{array}$ & $\begin{array}{r}2 \cdot 1 \\
47 \cdot 5 \\
24 \cdot 2 \\
26 \cdot 2\end{array}$ & $\begin{array}{r}1 \cdot 1 \\
46 \cdot 6 \\
28 \cdot 0 \\
24 \cdot 3\end{array}$ & & $\begin{array}{r}1 \cdot 0 \\
48 \cdot 7 \\
26 \cdot 1 \\
24 \cdot 2\end{array}$ & $\begin{array}{r}1 \cdot 0 \\
45 \cdot 6 \\
30 \cdot 3 \\
23 \cdot 1\end{array}$ & $\begin{array}{r}0 \cdot 7 \\
43 \cdot 8 \\
32 \cdot 7 \\
22 \cdot 8\end{array}$ & $\begin{array}{r}1 \cdot 3 \\
50 \cdot 6 \\
22 \cdot 6 \\
25 \cdot 5\end{array}$ & $\begin{array}{r}1 \cdot 2 \\
42 \cdot 0 \\
1.3 \\
55 \cdot 5\end{array}$ & $\begin{array}{r}0 \cdot 5 \\
46 \cdot 1 \\
1 \cdot 3 \\
52 \cdot 1\end{array}$ & \\
\hline 17. & $\begin{array}{r}23 \cdot 9 \\
1 \cdot 7 \\
8 \cdot 3 \\
66 \cdot 1\end{array}$ & $\begin{array}{r}5 \cdot 5 \\
3 \cdot 7 \\
48 \cdot 0 \\
41 \cdot 9\end{array}$ & $\begin{array}{r}2 \cdot 9 \\
48 \cdot 7 \\
1.0 \\
47 \cdot 4\end{array}$ & $\begin{array}{r}3 \cdot 7 \\
35 \cdot 1 \\
0 \cdot 7 \\
60 \cdot 5\end{array}$ & $\begin{array}{r}1.3 \\
43.8 \\
0.6 \\
54.3\end{array}$ & $\begin{array}{r}9.9 \\
28.8 \\
0.4 \\
60.9\end{array}$ & $\begin{array}{r}14 \cdot 3 \\
0 \cdot 8 \\
46 \cdot 4 \\
38 \cdot 5\end{array}$ & $\begin{array}{r}7 \cdot 8 \\
26 \cdot 5 \\
18 \cdot 3 \\
47 \cdot 4\end{array}$ & $\begin{array}{r}1 \cdot 6 \\
15 \cdot 8 \\
56 \cdot 4 \\
26 \cdot 2\end{array}$ & $\begin{array}{r}0.8 \\
19.5 \\
53.5 \\
26.2\end{array}$ & & & \\
\hline $17 \mathrm{a}$. & $\begin{array}{r}83 \cdot 1 \\
8 \cdot 1 \\
6 \cdot 5 \\
2 \cdot 3\end{array}$ & $\begin{array}{r}84 \cdot 4 \\
7 \cdot 5 \\
4 \cdot 5 \\
3.6\end{array}$ & $\begin{array}{r}83 \cdot 9 \\
7 \cdot 9 \\
0 \cdot 1 \\
8 \cdot 1\end{array}$ & $\begin{array}{r}76.2 \\
9.5 \\
5.5 \\
8.8\end{array}$ & $\begin{array}{r}80 \cdot 6 \\
8 \cdot 3 \\
0 \cdot 1 \\
11 \cdot 0\end{array}$ & $\begin{array}{r}86.9 \\
8.1 \\
0.2 \\
4.8\end{array}$ & $\begin{array}{r}89 \cdot 5 \\
7 \cdot 5 \\
0 \cdot 2 \\
2 \cdot 8\end{array}$ & & & & & & \\
\hline $17 \mathrm{~b}$. & $\begin{array}{r}11 \cdot 6 \\
19 \cdot 3 \\
2 \cdot 2 \\
66 \cdot 9\end{array}$ & $\begin{array}{r}26 \cdot 3 \\
2 \cdot 0 \\
14 \cdot 2 \\
57 \cdot 5\end{array}$ & $\begin{array}{r}21 \cdot 1 \\
21.6 \\
0.8 \\
56.5\end{array}$ & $\begin{array}{r}22.2 \\
25.1 \\
0.9 \\
51.8\end{array}$ & $\begin{array}{l}16 \cdot 8 \\
42 \cdot 1 \\
26 \cdot 1 \\
15 \cdot 0\end{array}$ & $\begin{array}{r}2 \cdot 5 \\
57 \cdot 7 \\
1.2 \\
38 \cdot 6\end{array}$ & $\begin{array}{r}3 \cdot 6 \\
9 \cdot 4 \\
32 \cdot 4 \\
54 \cdot 6\end{array}$ & $\begin{array}{r}2 \cdot 1 \\
19 \cdot 5 \\
32 \cdot 2 \\
46 \cdot 2\end{array}$ & $\begin{array}{r}1.5 \\
7.8 \\
39.5 \\
51.2\end{array}$ & $\begin{array}{r}0.5 \\
13.7 \\
1.1 \\
84.7\end{array}$ & $\begin{array}{r}1.0 \\
9.8 \\
38.2 \\
51.0\end{array}$ & $\begin{array}{r}1.3 \\
1.7 \\
60 \cdot 4 \\
36 \cdot 6\end{array}$ & $\begin{array}{r}1 \cdot 4 \\
24 \cdot 5 \\
1.4 \\
72 \cdot 7\end{array}$ \\
\hline $17 \mathrm{c}$. & $\begin{array}{r}22 \cdot 9 \\
3 \cdot 2 \\
14 \cdot 4 \\
59 \cdot 5\end{array}$ & $\begin{array}{r}18.9 \\
29 \cdot 0 \\
0.6 \\
51.5\end{array}$ & $\begin{array}{r}20 \cdot 1 \\
37 \cdot 0 \\
0.5 \\
42 \cdot 4\end{array}$ & $\begin{array}{r}14 \cdot 1 \\
32 \cdot 3 \\
1 \cdot 0 \\
52 \cdot 6\end{array}$ & $\begin{array}{r}13 \cdot 0 \\
31 \cdot 2 \\
0 \cdot 7 \\
55 \cdot 1\end{array}$ & $\begin{array}{r}14 \cdot 4 \\
31.6 \\
1.1 \\
52 \cdot 9\end{array}$ & $\begin{array}{r}2 \cdot 3 \\
10 \cdot 0 \\
61 \cdot 7 \\
26 \cdot 0\end{array}$ & $\begin{array}{r}0.9 \\
25 \cdot 1 \\
0.9 \\
73 \cdot 1\end{array}$ & $\begin{array}{r}1 \cdot 8 \\
23 \cdot 0 \\
17 \cdot 3 \\
57 \cdot 9\end{array}$ & $\begin{array}{l}22 \cdot 3 \\
24 \cdot 0 \\
16 \cdot 9 \\
36 \cdot 8\end{array}$ & $\begin{array}{r}15 \cdot 3 \\
27 \cdot 4 \\
2 \cdot 3 \\
55 \cdot 0\end{array}$ & & \\
\hline
\end{tabular}

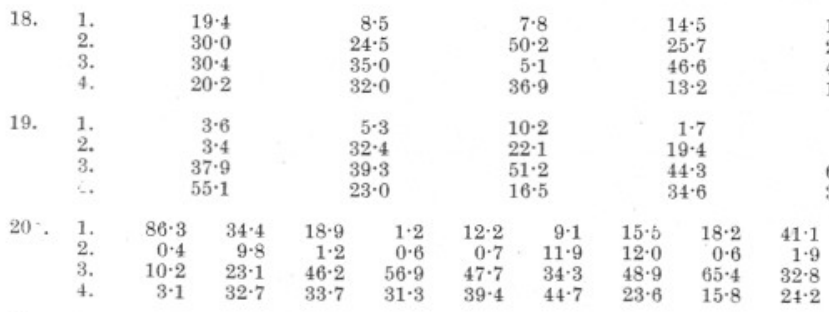

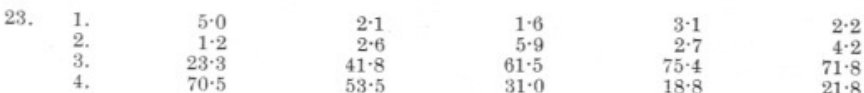

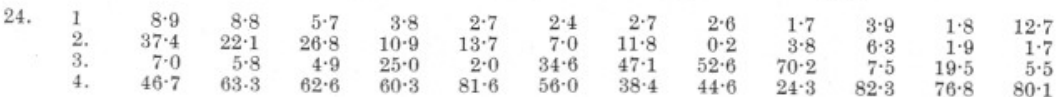

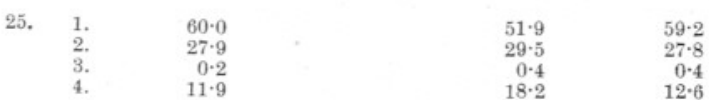

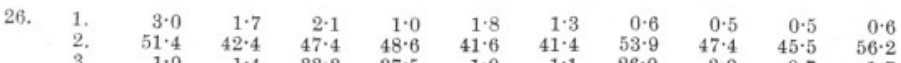

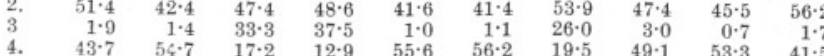


Pellets such as those of Calanus which fall in large quantities on the mud surface, but are more friable than those of Maldanids, are broken down in the course of the elutriation.

Figure 5 shows the distribution of these four grades for St. 16a in the Holy Loch as an example of the mid-loch type; while the figures for all the stations are given in Table VI. The material falling on the mud surface is largely fine detritus with some larger particles of organic origin : this is reflected in the high value of Grade IV at the surface. Much of this

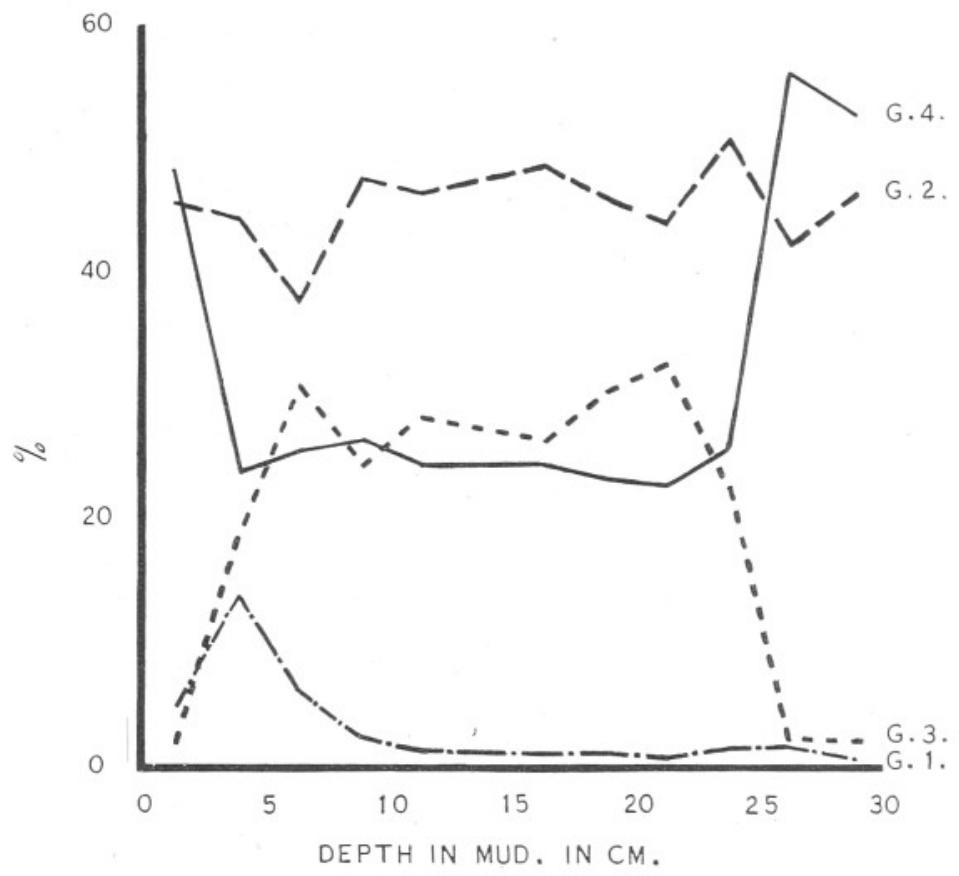

Fig. 5.-Distribution of particle grades with depth in the mud for St. 16a. G. 1 is the coarsest and G. 4 the finest.

fine detritus is eaten by animals living near the surface of the mud, and is converted by them into pellets. The effect of this is a rapid drop in the amount of Grade IV in the first 2 to $5 \mathrm{~cm}$. and a corresponding increase in Grade I. Grade II is more erratic, and shows no clear general rise to correspond with this, though such frequently takes place a few centimetres deeper. Below this depth the mud is packed to a much harder consistency with the result that it needs more rubbing down, and Grade I decreases rapidly. This fall then is assumed to be largely artificial. At the same time natural break down of the organic particles causes a rise in the amount of Grade III in the first 5 to $10 \mathrm{~cm}$. With the decrease in life 
deep in the mud, there is little further pellet formation, and Grade II remains more or less constant. But the break down of the organic particles is still continuing, and at a depth of about $25 \mathrm{~cm}$. Grade III suddenly drops to about $2 \%$ with a corresponding rise in the amount of Grade IV. The magnitude of this change may appear exaggerated since the difference in particle size in Grades III and IV is less than that between any other two grades. It points, however, to a steady slow break down of these organic particles.

As would be expected, at many stations conditions of deposition and change are not sufficiently regular to give as simple a curve as the one described. The type is best illustrated in the mid-loch stations, though the transitions in Grades III and IV usually take place more gradually than at St. 16a; Stations 8b, 11, 16a, and 23 show the type fairly well, and Stations 9, 13, and 13a less clearly. The loch-head type of station shows much more erratic results, though St. 14 suggests this type. The stations in the Dunoon Basin are, as in other characters, very erratic, and the sandy stations are of course of quite a different nature. Station 20a shows a high proportion of Grade I in the two sandy layers, with a low Grade I and a high Grade III and IV in the intermediate mud layers.

There are then two distinct processes taking place in the mud. Firstly, the fine material which falls on the surface of the mud is being eaten and converted into larger aggregates in the form of pellets which form quite the most outstanding feature of the muds; and secondly, there is a steady break down of the larger organic particles which fall on to the mud surface into finer material, many of these large particles being pellets, such as those of Calanus, but of a very much more friable nature than those formed in the mud. The pellet formation takes place only at the levels at which animal life exists, and chiefly close to the surface. Dr. Lloyd (4) has shown that bacteria are present in numbers in these muds in the deepest samples which have been taken, and perhaps due to them the break down of the organic particles continues to a greater depth.

\section{Nature and Rate of Sedimentation.}

With a view to determining the nature and origin of the sediments falling on the mud surface, and whether these showed variation in relation to any factors such as rainfall or plankton, collections have been made at various stations by means of two types of sediment pots.

The Type "A " pots are glass preserving jars, $32 \mathrm{~cm}$. high, with an internal diameter of $9 \mathrm{~cm}$. at the mouth. These were attached in string nets to a rope, buoyed at the surface. At the bottom of the rope was a lead weight, hanging just clear of the bottom at low water, and beyond this about 10 metres of slack rope attached to a heavy stone as an anchor. 
The pots thus moved vertically with the tide, but hung more or less upright.

Another type of pot, " B," was used later in order to ascertain whether there was any lifting of bottom material once it had settled on the mud. The gear consisted of a series of seven smaller pots attached at $30 \mathrm{~cm}$. intervals to a vertical iron rod, and this connected by a ring to a concrete block. A rope was attached to the top of the rod, and a set of submerged glass floats twenty metres above the rod kept the latter upright as well as keeping any slack rope clear of the pots; the floats were connected

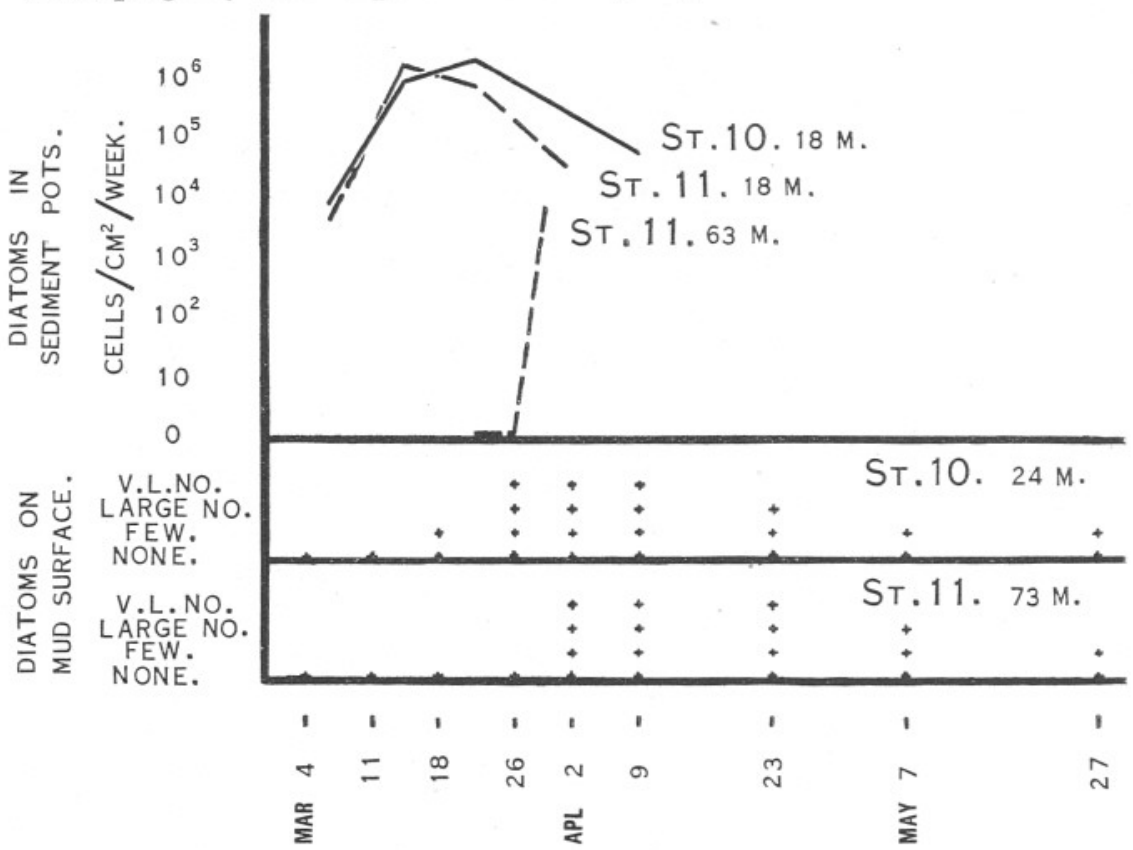

Fig. 6.-Occurrence of diatoms in the sediment pots (upper part of figure) and on the mud surface (lower part of figure) ; showing their appearance in sequence at the upper pots, the lower pots and the mud surface.

by a slack rope to a buoy at the surface. The pots used here were preserving jars, $17 \mathrm{~cm}$. high, with an internal diameter at the mouth of $5 \cdot 5 \mathrm{~cm}$.

Type "A" pots were put out first at Stations 10 and 11. At St. 10, at Loch Striven Head, where the depth of water is 24 metres, only one pot was attached at a depth of 18 metres. At St. 11, at the middle of the same loch, one pot was attached at 18 metres, and one just clear of the bottom at 68 metres, the depth of water being 73 metres.

The gear was first put out on February 25th, 1930, and the pots were replaced at first weekly, and later fortnightly. At the same time vertical 
hauls were made for plankton and tube samples of the bottom were taken for examination of the mud surface. Where possible diatom counts were made in the sediments by stirring up the latter with sea-water, and counting aliquot samples of it. The sediments were then washed, dried at $100^{\circ} \mathrm{C}$. and weighed. Some difficulty was experienced with the bottom pot at St. 11 touching the bottom, so that records for this pot were not obtained until March 18th. Also diatom counts in this pot later became impossible owing to the large numbers of fæcal pellets of Calanus which were present. Both the diatom numbers and the sediment weights are expressed as the amount falling on one square centimetre in one week. Since Skeletonema costatum formed $95 \%$ of the diatom population at this time, other forms were neglected in the counts.

At St. 11 the diatoms reached a maximum at the surface of the water on March 25th and about three days later at the 10 and 20-metre levels. In the sediments from St. 10 and the top pot of St. 11 the diatom numbers run a very similar course (Fig. 6), reaching maxima of two million at St. 10 and one million at St. 11 in the weeks March 11th-18th and 18th-25th respectively, and then decreasing again. At the same time a count in the bottom pot at St. 11 showed no diatoms for the week March 18th-25th, but 3,800 the following week. Although no actual count of the numbers of diatoms on the mud surface was possible, it was clear that they appeared there quite suddenly and were present for a time in enormous quantities. At St. 10 they appeared first on the mud surface in small numbers on March 18th, and were present in very great numbers the following week. At St. 11, in much deeper water, they were not found on the mud until April 2nd, when, as at St. 10, they were present in great quantity. They continued very numerous at St. 10 up to April 9th ; on the 23rd they

\section{TABLE VII.}

Numbers of Skeletonema Cells in Sediment Pots During 1930.

Expressed as number of cells per sq. cm. per week.

\begin{tabular}{|c|c|c|c|c|}
\hline March & $\begin{array}{c}\text { Date. } \\
\text { 4th-11th }\end{array}$ & $\begin{array}{l}\text { St. } 10 . \\
13,700\end{array}$ & $\begin{array}{l}\text { St. } 11 . \\
\text { Top Pot. } \\
11,500\end{array}$ & $\begin{array}{c}\text { St. II. } \\
\text { Bottom Pot. } \\
-\end{array}$ \\
\hline ," & 11th-18th & 987,000 & $1,040,000$ & - \\
\hline , & 18-26th . & $2,000,000$ & 669,000 & 0 \\
\hline , & 26th-April 2nd & - & 84,000 & 3,800 \\
\hline April : & 2nd-9th & 103,000 & - & - \\
\hline
\end{tabular}

were definitely less, and on May 7th there were few left. In the same way, on St. 11, they were still numerous up to April 23rd; on May 7th they were decreasing in numbers, and by the 27 th there were few left, though, 
of course, some are to be found on both stations all through the summer. The results are shown graphically in Figure 6 and the actual numbers in Table VII.

\section{TABLE VIII.}

Weights of Sediments in Sediment Pots During 1930. Expressed as mg. per sq. cm. per week.

Date.

February 25th-March 4th

March 4th-11th

,, 11th-18th

,. 18th-26th

, 26th-April 2nd

April 2nd-9th

,, 9th-23rd

,, 23rd-May 7th .

May 7 th-27th

,, 27th-June 14th
St. 10 .

$6 \cdot 9$

$4 \cdot 0$

$9 \cdot 4$

$9 \cdot 2$

$4 \cdot 2$

$7 \cdot 8$

-

$7 \cdot 1$

-
St. 11.

Top Pot.

$0 \cdot 54$

$0 \cdot 79$

$0 \cdot 71$

$2 \cdot 5$

$3 \cdot 4$

$4 \cdot 3$

$1 \cdot 0$

$1 \cdot 8$

$1 \cdot 1$

$0 \cdot 24$
St. 11.

Bottom Pot.

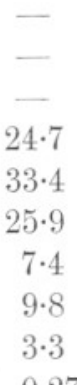

$0 \cdot 27$

The weekly weights of sediments for the three pots are shown in Figure 7, and the actual quantities in Table VIII. Those for the top pot at St. 11 show a strong peak following the diatom increase in the water, but about three weeks later; and the weight is greatly in excess of that which could be due alone to the diatoms in the pot. This excess is due to the presence of the pellets of Calanus finmarchicus of which the sediment was just then almost entirely composed. The vertical tow-nettings showed that these copepods began to appear in large numbers just after the spring diatom increase; and at the same time their pellets first occurred in the pots. The suddenness of the drop in sediment weight after April 9th suggests that the Calanus shoals were no longer to any great extent feeding - or at any rate shedding their pellets - at this level in this particular area. The weights of sediment for the bottom pot at this station follow a similar course, with a peak following the spring diatom increase maximum, though for no obvious reason the peak comes a week earlier in the case of the bottom pot. During the "Peak," the actual weight of the sediment is considerably greater at the bottom than in the upper pot, but later in the season they show little difference, i.e. $0 \cdot 24 \mathrm{mg}$. in the upper pot, and $0 \cdot 27 \mathrm{mg}$. in the lower. The pellets in the pots show that the difference is due, at any rate largely, to swarms of Calanus and Euphausids.

The constitution of the sediment is very similar in the two pots. In both there is a considerable amount of fine detritus of unrecognisable origin, although much of it clearly comes from the plankton; a few 
quartz grains up to $0 \cdot 2 \mathrm{~mm}$. in length, though usually smaller, may be present. The main bulk of the material in both pots during the summer is pellets, those of Calanus being predominant in the top pot and mixed with the larger pellets of Mysids and Euphausids in the lower pot. There are always a certain number of dead animals from the plankton, i.e.
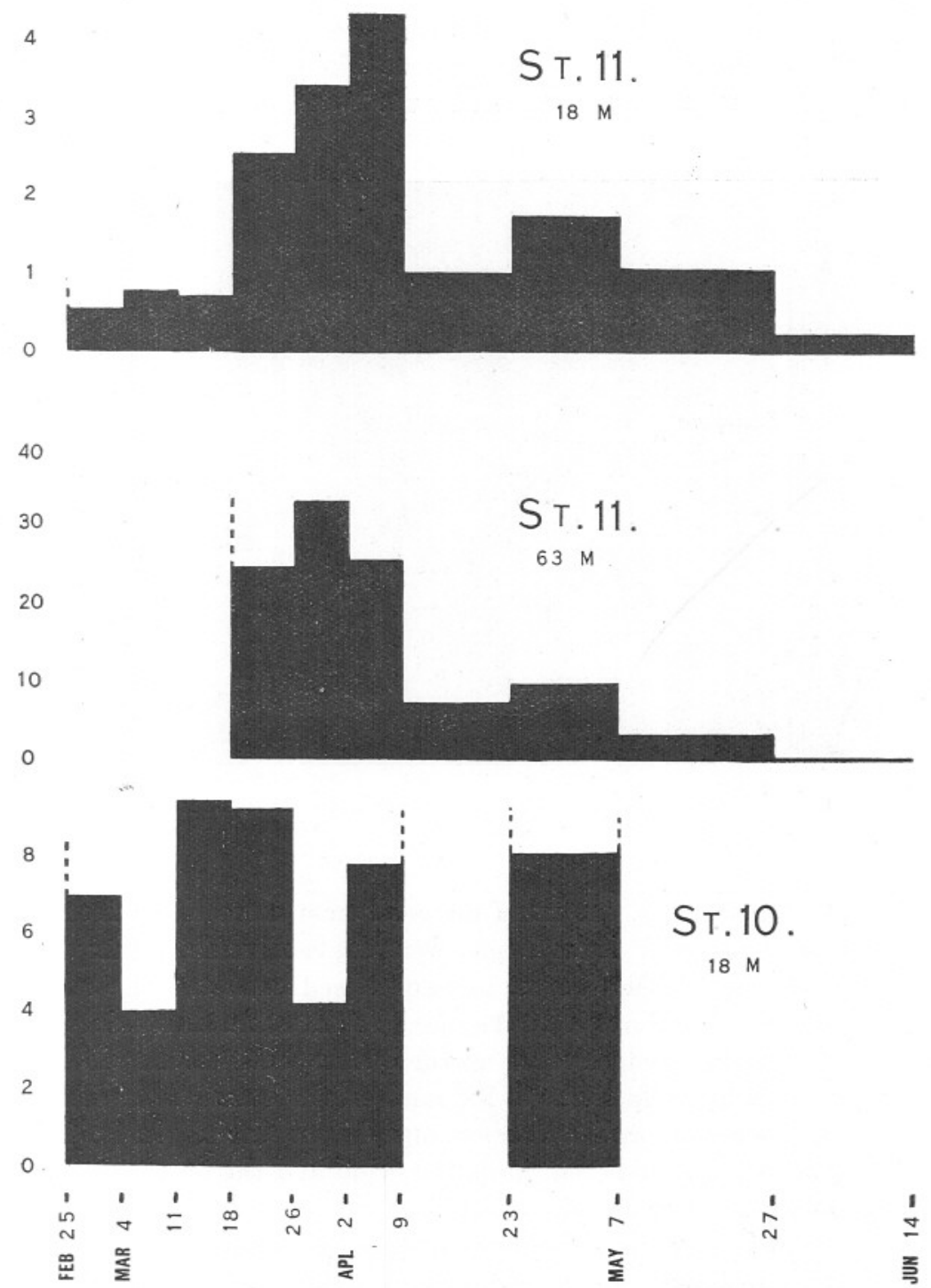

FIG. 7.-Weights of sediments - in mg. per sq. em.-_collected in Loch Striven during, the period of the spring diatom increase. Note the vertical scale is different in each example. 
copepods, rotifers, etc., as well as diatoms, the latter sometimes in very great quantity. At certain seasons, too, young lamellibranchs and polychætes are very common in the pots.

The sediment weights from the pot at St. 10 are very much more erratic than those from the other station, and show no "Peak" correlated with the spring diatom increase. In general the weight is considerably higher than that for the pot at the same depth at St. 11, and the sediment generally contains much more sand. Since we know that the contribution to it from the plankton, as reflected by the numbers of diatoms, is very

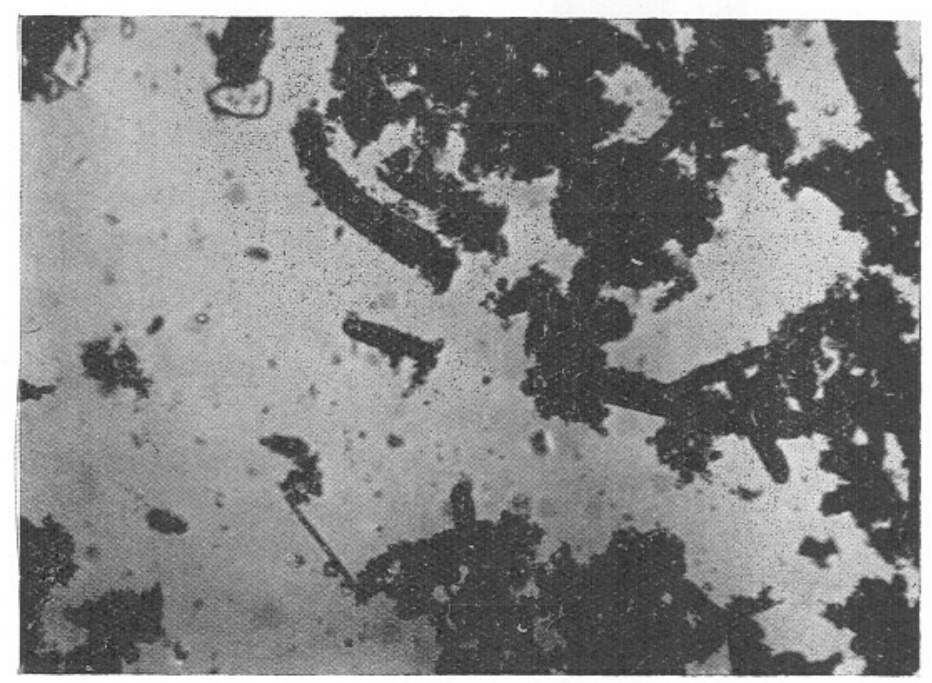

FIG. 8.-Sediment from St. 17d, showing Calanus fæces, diatoms and sand grains.

similar on both stations, the extra material must come either from the land or from the beach. If the former were the case we should expect to find some correlation between the amount of sediment and the rainfall and this has been found not to be the case. At the same time it does bear some relation to the strength of the northerly component of the wind, and it appears that this wind tends to lift sand off the banks at the sides of the loch in this region, and to carry it into deeper water. On St. 11 no such relation is found between rainfall or wind and the amount of sediment or the occurrence of sand grains in it.

In order to compare these results with those for less sheltered positions, two other stations were chosen, and pots put out at each of these for two periods of over a week. The first position chosen was St. 17d in Kilchattan Bay. The depth here is 22 metres, and the bottom sandy and 
steeply shelving, and the bay itself fairly sheltered. One pot was placed as before at a depth of 18 metres. The weights of sediment per $\mathrm{cm} .{ }^{2}$ per week were as follows :-

$$
\begin{aligned}
& \text { May 22nd to June 5th ․ . . } \quad 3.42 \mathrm{mg} \text {. } \\
& \text { June 5th to June 14th ․ . . } \quad 5 \cdot 37 \mathrm{mg} \text {. }
\end{aligned}
$$

The nature of the sediment was very similar to that obtained at this time in the lochs, i.e. mainly Calanus pellets together with some Euphausid pellets, and a few diatom chains. There was also a small amount of fine detritus and some sand grains up to $0.5 \mathrm{~mm}$. in length, these being more abundant during the second period: one of these sediments is shown in Figure 8 . The greater amount of sediment in this second period was due to the drifting of sand particles offshore, the wind being almost continuously from the east for the first period, and from the north-west for the second.

The pots were then put down at the same depth at St. 1c off Fairlie sands. The bottom here is sandy and exposed to a strong tide, and inshore there are extensive beds of Zostera. The first time there was a small whiting in the pot, so that the results may be too low. The material was almost entirely sand, together with some Calanus pellets. Some fragments of decayed Zostera were present, but these were very few considering the extent of the Zostera beds close to the pots, and as in the case of the Danish waters the weed is no doubt being carried in large masses into deeper and quieter water before it settles (Ostenfeld, 8). The weight of sediment per cm. ${ }^{2}$ per week was $20.3 \mathrm{mg}$. The following week the Type "B" pots were used, and showed very similar results, with evidence that much of the material was being swept from the inshore sandbanks, there being pellets of Nucula in the pot $90 \mathrm{~cm}$. above the bottom, and much sand in all pots. The average weight of sediment for the pots was $33 \cdot 3 \mathrm{mg}$.

The Type "B" pots were also used on St. 11 in Loch Striven to ascertain whether there was any lifting of bottom material once it had been deposited. Their evidence is not conclusive, but it may be inferred from it, together with the presence of undisturbed diatoms on the extreme surface after the spring increase and also from the evidence of layering which is discussed later, that no such lifting takes place in these sheltered waters.

The results from these pots for a typical period are given below; the top of No. 1 pot lying about $20 \mathrm{~cm}$. above the mud surface, and that of No. 7 pot about $230 \mathrm{~cm}$. above it.
Wt. of Sediment in $\mathrm{mg} . \quad 1$.
per $\mathrm{cm} .^{2}$ per week $\quad \cdot 0 \cdot 72$
$2 . \quad 3$.
4.
5. 6.
7.

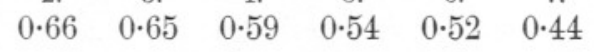


In the bottom pot-No. 1-there were a few Maldanid pellets indicating slight disturbance of the bottom in lowering the gear, but this did not affect any of the higher pots. The material in the latter was partly fine detritus, but chiefly pellets; these were mostly from Calanus in the top pot-No. 7-but with a great many large Euphausid pellets in the lower pots. Although no count was possible, the number of these seemed sufficient to account for the increase in weight near the bottom, where the Euphausids are most abundant. If any lifting of bottom material had taken place, recognisable, small bottom pellets would have been expected at any rate in No. 2 pot and none were found.

It is interesting to compare these results with those given by Petersen for Thirsted Bredning in 1910 (Petersen, 9). These give an average of $6 \mathrm{mg}$. of sediment per sq. cm. per week; and if we neglect the fourth, which was taken in much shallower water than the others, they give a striking suggestion of a spring maximum similar to that found in Loch Striven. Petersen states, however, that the sediment collected consisted almost entirely of fine detritus of unrecognisable origin, and that the plankton contributed little to it.

\begin{tabular}{|c|c|c|c|c|c|}
\hline \multicolumn{6}{|r|}{ in $\mathrm{mg}$. per sq. cm } \\
\hline \multirow{2}{*}{$\begin{array}{l}\text { No. } \\
1 .\end{array}$} & \multicolumn{2}{|l|}{ Date. } & & Depth in $\mathrm{m}$. & per week. \\
\hline & April 1st to April 15th. & . & . & 12 & $1 \cdot 36$ \\
\hline 2. & April 15th to April 22nd & . & . & 12 & $3 \cdot 51$ \\
\hline 3. & April 25th to May 21st. & . & . & 14 & $15 \cdot 68$ \\
\hline 4. & June 4th to July 5th . & . & . & 7 & $(0 \cdot 49)$ \\
\hline 5. & July 6th to August 10th & . & . & $10 \cdot 5$ & $8 \cdot 96$ \\
\hline
\end{tabular}

\section{Estimation of Rate of Deposition of Sediments.}

If a tube sample of mud from a suitable locality is allowed to stand in a bright light or even in direct sunlight, it begins after a few weeks to develop alternate bands respectively lighter and darker than the original colour of the sample, and these may continue to intensify for from one to eighteen months. No such bands are visible in the fresh sample. While the light bands vary from 3 to $7 \mathrm{~mm}$. in depth, the dark bands are narrow and not more than 1 or $2 \mathrm{~mm}$. deep, and the pigmentation appears to be due to the formation of peat (see below). From a series of samples from St. 11, of which three, taken in February, early June, and July, are shown in Figure 9, it is clear that the dark band represents the sediment laid down at about the period of the spring diatom increase, and it is most probable that the colouration is due to the diatoms. At any rate it was shown in the previous section that there is a rich layer of diatoms on the surface of the mud on this station during the whole of April, and it is about this time that a new dark band appears. 
No trace of diatom skeletons can be found in the layers, but they are probably an unstable form of silica which quickly disappears. The peat formation is probably associated with the very large numbers of bacteria
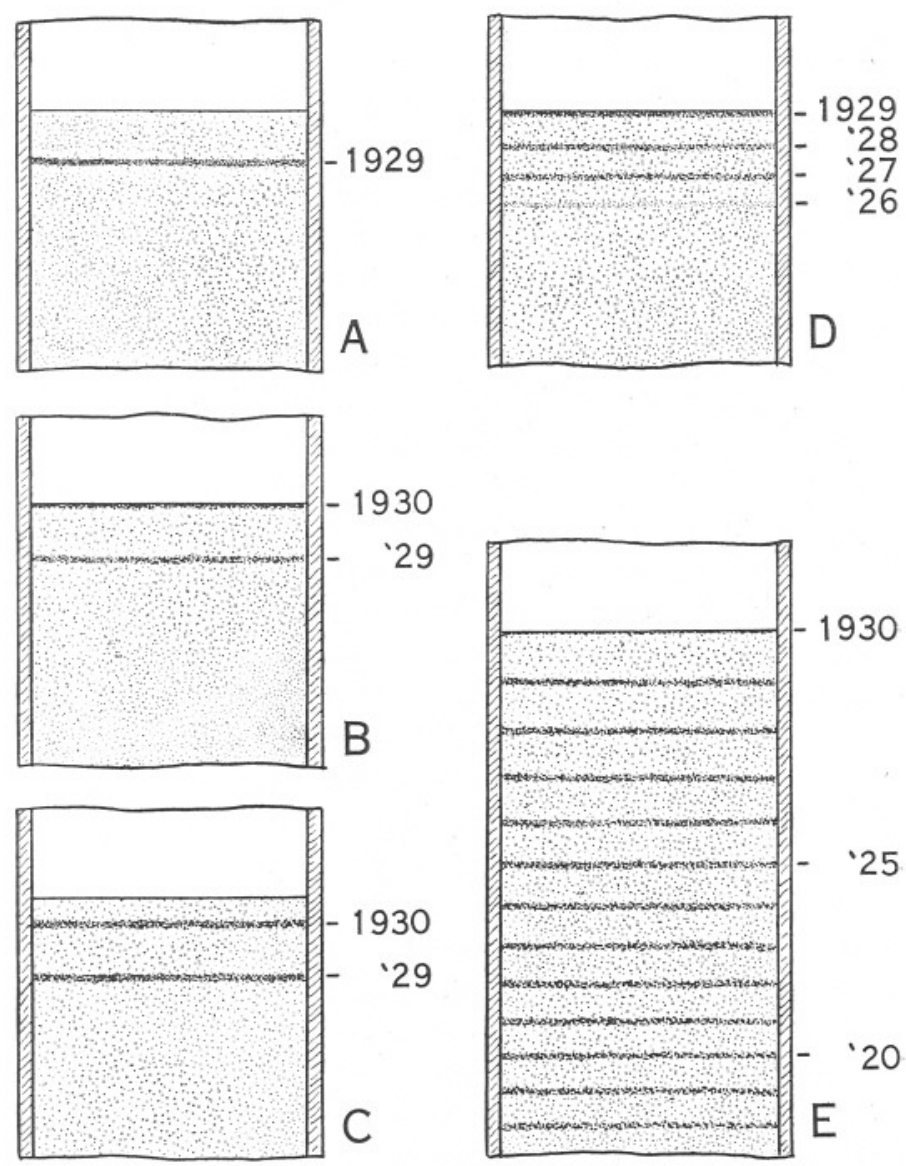

Fig. 9.-Annual layers as seen in tube samples of mud. The dark layers. correspond to the spring diatom increase.
A. St. 11. 11th February, 1930.
B. St. 11. 5th June, 1930.
C. St. 11. 18th July, 1930.
D. St. 7. May, 1929.
E. Theoretical spacing of undisturbed bands for St. 11 ; packing practically ceases after ten years.

found by Lloyd (4) on the mud surface during the period of this rain of diatoms.

These bands are only found on stations where there is very fine sediment. falling, and where there are no currents to disturb this once it has settled. 
They are, therefore, not to be seen in the coarse mud of the Loch Head stations, nor those in the region of the Dunoon Basin where there are strong tidal currents, but they are well seen in the deeper ones at Stations $11,7 \mathrm{a}$, and $7 \mathrm{~b}$. On St. 11 the maximum number of bands which has been found is two, but on St. 7 four have been seen, the deepest rather faint. The non-appearance of bands below this is in all probability due to the mixing of the mud by the fauna, and this is in accord with the fact that on both stations the dominant forms, i.e. nematodes, are most abundant in the 1 to $2 \mathrm{~cm}$. layer, while the layering is best seen in the top centimetre.

From the distance between the layers, it is possible to calculate the rate at which mud is being laid down at any station. For St. 11, the density and water content of the mud, being known, a graph can be obtained, giving the rate of packing of the mud with depth; and from this, the theoretical spacing of the layers shown in Figure 8, E. At the surface the layers are $6 \mathrm{~mm}$. thick, at about $5 \mathrm{~cm}$. they have packed to $4 \mathrm{~mm}$. thick, and beyond this depth there is little further packing. The top $5 \mathrm{~cm}$. then corresponds to $9 \frac{1}{2}$ years' sedimentation, and below this $40 \mathrm{~cm}$. corresponds to about 100 years. At St. 7 the layers are $3.5 \mathrm{~mm}$. apart at the surface so the rate of sedimentation is a little more than half that at St. 11.

The evidence that these are annual layers seems sufficient, but their spacing may be checked by comparison with the results from the sediment pots. If the water content at the surface of St. 11 is $85 \%$ and the density $2 \cdot 3$, the amount of material between the first two layers corresponds to $207 \mathrm{mg}$. per sq. cm. or a weekly average of about $4 \mathrm{mg}$. This is in agreement with the results from the bottom pot at the same place, which collected $100 \mathrm{mg}$. of sediment per sq. cm. during the 12 weeks which included the spring increase, or a weekly average of $8.3 \mathrm{mg}$. Considering the abnormally high values obtaining at the time, this is quite reasonable.

A similar type of stratification is found in the muds on the bottom of the Black Sea, although the conditions of deposition there are very different. The following is an extract (translated) from a paper by Schokalsky (10) in which he shows that the layers are probably the result of an alternation of a winter terrigenous deposit with a spring and summer planktonic deposit. Also, in the Black Sea muds, the sediment is undisturbed from the time that it is deposited, so that it is possible to trace layers layed down seven thousand years ago in the very long cores which he has taken.

"The fine stratification gives ten to twelve layers per millimetre; it shows them best in samples taken near the coast. Each of the fine layers is composed of two, one black and organic in nature, the other grey and inorganic; and we have likened each layer to an annual deposit. The black is the product of the plankton dying in winter, while the grey is the accumulation of debris carried down by rivers and coastal waters 
in the spring. If we accept this hypothesis we arrive at the following results: a thickness of one centimetre is laid down in 50 years. In the layers of calcareous mud a similar stratification is observed, each layer again being double, one formed of calcium carbonate and the other of dead organic matter. From this we conclude that in any case the deposition of a column of mud of one metre will take about 4000 years."

I am indebted to Mr. A. Halimond for the following report on the geological nature of the layered Clyde Muds.

"Sections prepared by infiltration with artificial resin show a typical fine grained mud with small angular quartz grains and flakes of mica in a clayey ground mass. Small brown pellets are common, and there are also larger circular and elliptic areas containing in the centre the same minerals as the mud, but richer towards the margin in an isotropic brownish material, probably phosphate. A section through the top centimetre of the mud (E. 27845) shows a clear mud next to the surface, while at a depth. of about half a centimetre there occurs a layer containing particles of brown amorphous ground mass which are probably either peaty or phosphatic matter.

At a depth of nine centimetres (E. 27844) the mud is generally similar to the lower layers of the surface."

The circular and elliptical bodies referred to above are, of course, the fæcal pellets of Maldanid worms.

\section{Distribution of Fauna.}

Although the sea bottom of the Clyde Sea Area has been very thoroughly worked from a faunistic point of view, such quantitative work as has been done has all been restricted to the shore and immediately below low-tide mark, and even here only horizontal and not vertical distribution in the substratum has been described in detail. In the deep water, the fauna may be divided into two main groups. The first comprises the large forms which either live at the surface of the mud, or can come up to it at intervals to breathe, and also the burrowing and tubicolous forms which, while penetrating to a considerable depth, maintain an open connexion with the overlying water. Of this type we have taken Calocaris macandrea in its burrow at a depth of $20 \mathrm{~cm}$., and tubes of Sabellid worms extending to a depth of over $30 \mathrm{~cm}$. To take these in quantity, however, an instrument holding a large amount of mud would be necessary, and this has not been available.

The second group comprises those forms sufficiently small to be contained in a layer of mud not more than about a centimetre thick, and whose environment is determined by the nature of this layer. Since the nature of the mud may change very considerably within a vertical space of two 
centimetres, it is necessary to examine only forms of less than about five millimetres long, if they are to be correlated with the nature of the mud in particular layers.

Several tube samples were taken from each station, and the mud cut up into one-centimetre layers as soon as possible. This was then passed successively through sieves of 10 and 120 meshes to the inch, and any polychætes retained in the former were neglected; small nematodes were also retained sometimes, entangled in masses of detritus, and these were removed and counted. The whole of the material left in the 120mesh sieve was examined under a binocular microscope, and the various living animals picked out. The results of counts on a Loch Head, a MidLoch, and a Deep Water Station are given in Table IX, and the distribution of the two commonest forms, namely nematodes and copepods, is

ST. 10.

LOCH HEAD

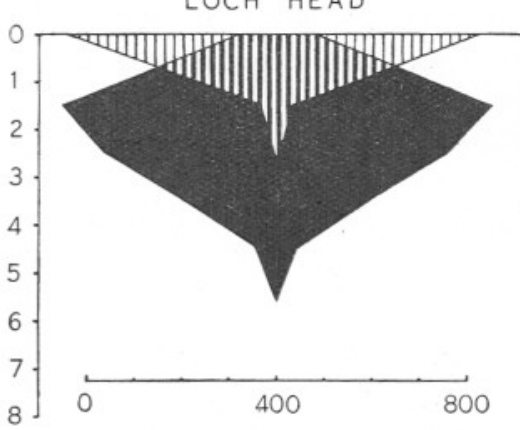

ST. 11 . MID LOCH.

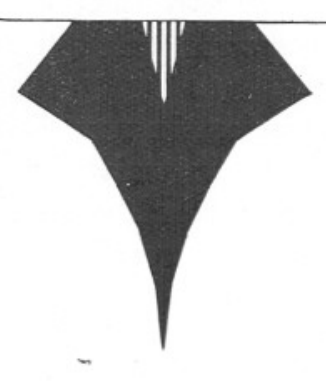

ST, 7ㄹ DEEP WATER.

FIG. 10.-Distribution of the smaller fauna with depth in the mud on three types of stations: numbers of individuals - nematodes (black) and copepods (shaded) - in each one-cm. layer in a column of mud of $100 \mathrm{~cm} .^{2}$ surface area.

shown in Figure 10. Except in the surface layer, only very few ostracods and copepods were found, and these few individuals may either have straggled down from the upper layers, or else have been carried down into a deeper layer by the rim of the sampler. At any rate, they occur too rarely in the deeper layers to show definitely that they live there, and for this reason they have been neglected in Figure 10. The polychætes taken were mostly immature forms, and from their patchy distribution are not suitable for comparison.

All the copepods taken were Harpacticids, and they were limited almost entirely to the surface centimetre of the mud. Mr. Elmhirst examined a sample of these and found them to consist chiefly of Danielssenia typica Boek. and Cletodes longicaudatas Boek., with a few Harpacticus uniremis Kroyer. They are also very much more abundant in the Loch Head type of station than in either of those in deeper water, 
the former showing a total density of 697 copepods per $100 \mathrm{sq}$. cm. while the other two show only 106 and 136. Ostracods show a very similar range, being also restricted chiefly to the top centimetre, and decreasing in abundance in the deeper stations, the total numbers per hundred square centimetres being respectively 125, 86, and 12 .

The nematodes, on the other hand, show an entirely different type of distribution. Their maximum numbers in each station are attained in the 1-2-cm. layer and they are still found in quantity two centimetres deeper. While, as in the case of the copepods, the total densities decrease

\section{TABLE IX.}

\section{Fauna Densities.}

Numbers per $100 \mathrm{sq}$. cm. in each $1 . \mathrm{cm}$. layer.

\begin{tabular}{|c|c|c|c|c|c|c|c|c|c|c|c|c|}
\hline \multirow[b]{2}{*}{$\begin{array}{l}\text { Depth } \\
\text { in mud } \\
\text { in } \mathrm{cm} .\end{array}$} & \multicolumn{4}{|c|}{$\begin{array}{c}\text { Loch Head Type } \\
\text { St. } 10 .\end{array}$} & \multicolumn{4}{|c|}{$\begin{array}{l}\text { Mid-Loch Type } \\
\text { St. } 11 \text {. }\end{array}$} & \multicolumn{4}{|c|}{$\begin{array}{l}\text { Deep Water Type } \\
\text { St. 7B. }\end{array}$} \\
\hline & 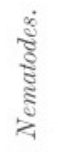 & 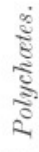 & 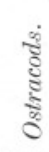 & $\begin{array}{l}\text { हैँ } \\
\text { ईँ } \\
\text { है }\end{array}$ & 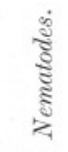 & 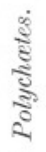 & $\frac{\dot{\delta}}{\tilde{\delta}}$ & 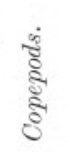 & 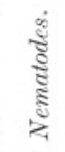 & 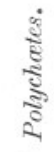 & $\frac{\dot{s}}{\tilde{\delta}}$ & 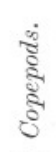 \\
\hline $0-1$ & 402 & 25 & 106 & 607 & 452 & 21 & 58 & 62 & 341 & 19 & 12 & 93 \\
\hline $1-2$ & 900 & 3 & 16 & 68 & 615 & 25 & 12 & 12 & 459 & 0 & 0 & 6 \\
\hline $2-3$ & 728 & 3 & 3 & 16 & 302 & 29 & 4 & 0 & 341 & 12 & 0 & (19) \\
\hline $3-4$ & 395 & 3 & 0 & 6 & 195 & 4 & 4 & (4) & 218 & 12 & 0 & (6) \\
\hline $4-5$ & 84 & 0 & 0 & 0 & 95 & 8 & 4 & (12) & 87 & 0 & ( & (12) \\
\hline $5-6$ & 6 & 0 & 0 & 0 & 33 & 0 & 0 & (8) & 81 & 6 & ( & 0 \\
\hline $6-7$ & 0 & 0 & 0 & 0 & 12 & 0 & 4 & (8) & 37 & 0 & 0 & 0 \\
\hline $7-8$ & - & - & - & - & - & - & - & - & 6 & 0 & 0 & 0 \\
\hline $8-9$ & - & 一 & - & - & - & - & - & - & 0 & 0 & 0 & 0 \\
\hline $9-10$ & - & - & - & - & 一 & - & - & - & - & - & - & - \\
\hline $10-11$ & - & - & - & - & 0 & 0 & 0 & 0 & - & - & - & - \\
\hline Total & 2515 & 34 & 125 & 697 & 1704 & 87 & 86 & 106 & 1570 & 49 & 12 & 136 \\
\hline
\end{tabular}

with increasing depth of water-namely, 2515, 1704, and 1570 respectively per hundred square centimetres - the depth in the mud to which they are taken increases in the deeper stations, the limits for the three stations being 6,7 , and $8 \mathrm{~cm}$.

From the general similarity of the three types of station, together with the decrease in total densities of all forms in the deeper water stations, it would appear that either there is less available food in the deeper water or else other conditions are generally less favourable there. The chief differences between the three stations are the steadily decreasing rates of sedimentation with increasing depth of water, and hence a decrease in available food, and the much smaller oxygen avidity at the surface in St. 10. Now in all the muds, even at the surface, the oxygen content is zero, and for forms needing free oxygen this must necessarily be a limiting 
factor. In view then of the experiments described below it is clear that the copepods cannot live for long in the absence of oxygen, and hence must live within easy reach of the oxygenated overlying water. The nematodes on the other hand are able to live in the absence of oxygen, at any rate for a longer period, so that the deeper layers, where they will have little competition, are available to them. Another factor, however, which must not be forgotten is the consistency of the mud, for it is clear that a copepod will find it difficult to move in a clayey mud, though able to do

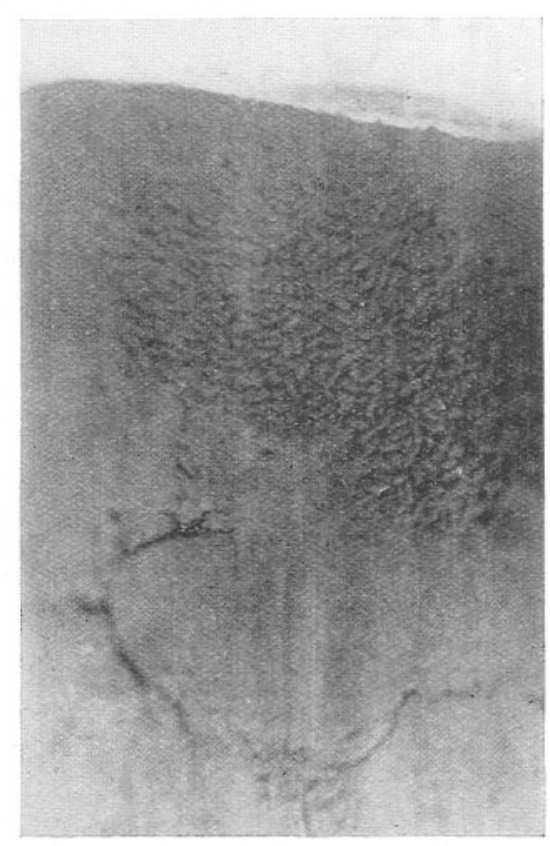

FIG. 11.-Photograph showing mud converted into pellets by a Maldanid worm.

so easily in the loose surface mud, while a nematode from its shape and size is well adapted for movement even in the stiffer clays.

The reaction of the mud cannot be to any extent a limiting factor, since the differences found are so small, and also the differences between two stations in which the same species may occur are greater than those occurring within any one of the stations.

The larger forms are not taken in the sampler in sufficient numbers to allow any quantitative estimate of their importance. But they have a very great effect on the structure of the mud, since most of them feed on the finer particles either in the mud itself or falling from the water, and convert them in their guts into firm pellets. That this process is of extreme 
importance in the muds is shown by the large numbers of pellets present. At St. 11, for example, $30 \%$ to $50 \%$ of the mud is converted into these pellets, and the average of a series of counts shows about 3400 pellets per cubic centimetre of mud. The effect of this aggregation on the effective particle size of the mud is obvious. In the first place, the liability of the mud to be carried by currents is greatly decreased, and in the second place, the mud is kept very much more open so that water can more readily diffuse into it. This will be clearly seen from an examination of Figure 11, although this certainly represents an abnormal sample in which practically the whole of the mud had been converted by a Maldanid worm into pellets. From a biological aspect also the process is important, since mud which has once passed through an alimentary canal and presumably lost much of its available nutrient material, instead of being set free to be re-eaten by some other animal is bound up in a distinctive form which will be refused by another selective feeder meeting it. This can be clearly seen in the case of a form like Syndosmya alba which lies buried, while its extended siphons feed indiscriminately from the surface of the mud. Periodically it blows out rejected material through one siphon, and this is found on examination to be largely composed of the pellets of other molluses, worms, etc. The method of identification of these pellets, with special reference to those occurring on St. 11, is given in another paper (Moore, $\mathbf{y}$ ). While accurate counts of the numbers of pellets are difficult, the following very approximate figures show the dominant form on St. 11.

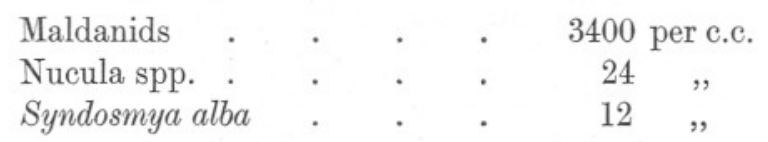

These results also demonstrate the value of pellet determinations compared with sampling of the actual animal by a " mud bucket" since the latter takes the two molluscs readily, but only occasional fragments of the deeper burrowing Maldanids.

\section{Resistance to Anærobic Conditions.}

In order to determine the ability of various of the mud-dwelling forms to exist in water containing no oxygen, the following tests were made. Animals were kept in the dark in stoppered bottles, in water from which as much oxygen as possible had been removed. This was done by alternately removing dissolved gas with a pump, and then saturating with washed hydrogen, and the oxygen content as determined by Winkler's method was reduced to less than $0 \cdot 2 \mathrm{mg}$. per litre. In some cases this residue of oxygen also was removed by shaking with a small quantity of mud, but with no difference in results. 


\section{Syndosmya alba.}

None lived for more than three and a half days, even in large vessels which were stirred twice a day with a stream of hydrogen. This is to be expected, since they normally keep a continuous connection with the overlying water by means of their siphons.

\section{Nucula tenuis.}

These lived and remained active for periods of from five to seventeen days, and death then may have been due to poisoning of the water by the animals' own excreta. The results are in agreement with the fact that Nucula is not provided with siphons like Syndosmya, and yet is found feeding at a depth of several inches below the surface of the mud.

\section{Copepods.}

Seventy-five per cent were dead at the end of 7 hours, and $90 \%$ at the end of 14 hours. A tube full of copepods in which all were apparently dead was opened at the end of 12 hours, and on exposure to the air, about two-thirds of them recovered, but after a longer period without oxygen there was no recovery.

\section{Nematodes.}

At the end of nine days $75 \%$ were dead and $90 \%$ at the end of a fortnight, while all the controls in oxygenated water were alive.

In these experiments the animals are undoubtedly in an environment very different from that of the mud, so that it is not legitimate to infer from them more than the fact that Syndosmya and copepods cannot live long in the absence of oxygen, but Nucula and the nematodes in question may be able to withstand it for comparatively long periods. In the case of the latter it would seem possible that they might live permanently without free oxygen. In an attempt more closely to reproduce natural conditions in the mud, the water in the tubes above a fresh sample of mud was replaced by deoxygenated water. The tube was then sealed at the bottom with paraffin wax, and the water covered with a layer of liquid paraffin. The tube was stored in the dark, and opened after thirty-five days. At the end of this time all the copepods were dead, but the nematodes were normal and active.

\section{SUMMARY OF GENERAL CONDITIONS IN THE MUD.}

The deep channels throughout the area have in general a bottom oomposed of mud. Where the bottom is sandy this is usually found to be associated with strong currents. The typical muds show a gradation from the type found at the heads of the lochs where streams enter, and the water is shallow, to those found in deeper water. 
In all there is a steady deposition of fine material, the source of a large proportion of which being referable to the plankton in the overlying water. The chief differences in the material of the muds lie in the finer sediments in deeper water, and the coarser ones near the loch-heads, where large particles are contributed both by streams and by the beds of littoral and sublittoral algæ. The particles other than these vegetable remains reach the bottom in the form of fine detritus, diatoms, etc., and the fæcal pellets of forms like Calanus and various Euphausids; and at different seasons the proportions of these vary, giving rise to very different types of sediment.

In these sheltered channels the surface of the mud appears to be sharply marked off from the overlying water, there being normally no mixing of the mud into the water. But the mud near the surface is in a very soft condition with a high water content of $80 \%$ to $85 \%$. This soft condition may extend to a depth of 5 centimetres or more, representing about ten years' deposition, but by various agencies water is being pressed out of the mud, bringing it to a more clayey constitution, and at a depth of 20 centimetres it is usually stiff and comparatively hard. The midloch and deep-water types of station are alike in this, but the loch-head ones differ in that the mud is of a more open nature owing to the large organic particles present, and often also to an admixture of sand grains.

The nature of the mud changes considerably with increasing depth. The particles themselves are no longer the same. The friable pellets of the planktonic organisms are no longer recognisable and have probably broken down. At the same time much of the fine material has been aggregated by the various mud-dwelling forms into firm pellets which may last at any rate for a hundred years and probably for much longer. The chemical nature of the mud is also changing; the total nitrogen and phosphate decreasing as well as the soluble phosphate, the latter decreasing most rapidly in the top five centimetres where most water is being expressed. Of special biological importance is the complete absence of oxygen even in the surface layers of the mud. The variation of $\mathrm{pH}$ is not great and has not been correlated with any other factors.

The relation of these factors has been studied chiefly with regard to the smaller organisms of which Copepods and Ostracods especially, and also Nematodes, are more abundant on the shallower stations. The distribution of all forms with depth in the mud seems to be regulated chiefly by their need for free oxygen and their mechanism for obtaining it. Syndosmya can burrow to the depth of its siphons, but dies quickly in the absence of oxygen. Nucula has no such siphons, but on the other hand is able to resist anærobic conditions of much longer periods, and can burrow to a considerable depth. Of the smaller forms with no such mechanism as that of Syndosmya, the various nematodes found are able, 
like Nucula, to resist anærobic conditions for some time if not permanently, while the small crustacea, of which Harpacticids are the most abundant, have no such resistance, and are therefore confined to the extreme surface layers. Dr. Lloyd (4) has shown that bacterial life is abundant deep in the muds, but with the exception of tubicolous or burrowing forms nearly all animal life ceases at about ten centimetres.

Finally, I wish to express my thanks to the members of the staff at Millport who have assisted in the work.

\section{REFERENCES.}

1. Atrins, W. R. G. The Phosphate Content of Fresh and Salt Waters: in its relationship to the Growth of Algal Plankton. Journ. Mar. Biol. Assoc., N.S., Vol. XIII, 1923, p. 119.

2. ClaRK, W. M. The determination of hydrogen ions. Baillière, Tindale and Cox, London, 1928.

3. International Society of Soil Science. Commission 2. Leningrad, July 1930. Report of the Committee on Soil Measurements. as adopted at the Second International Soil Congress.

4. Lloyd, B. Muds of the Clyde Sea Area, II. Journ. Mar. Biol. Assoc., N.S., Vol. XVII, No. 2, 1931.

5. Moore, H. B. The Muds of the Clyde Sea Area, I. Phosphate and Nitrogen Content. Journ. Mar. Biol. Assoc., N.S., Vol. XVI, No. 2, 1930.

6. Moore, H. B., and Neill, R. G. An Instrument for Sampling. Marine Muds. Journ. Mar. Biol. Assoc., N.S., Vol. XVI, No. 2. 1930.

7. Moore, H. B. The specific identification of Fæcal Pellets. Journ. Mar. Biol. Assoc., N.S., Vol. XVII, No. 2, 1931.

8. Ostenfeld, C. H. Report Danish Biol. Station. XVI, 1908.

9. Petersen, C. G. J. Valuation of the sea. I. Animal life on the sea: bottom; its food and quantity. Rept. Danish Biol. Station. XX, 1911.

10. Schokalsky, J. L'Expédition océanographique de la Mer Noire. C. R. Acad. Sci. Paris, CLXXXVI, 1928, pp. 1707-1709. 